\title{
Nursing home-sensitive conditions: analysis of routine health insurance data and modified Delphi analysis of potentially
}

\section{avoidable hospitalizations [version 1; peer review: 1 approved,}

\section{1 approved with reservations]}

\author{
Sabine Bohnet-Joschko(D1, Maria Paula Valk-Draad (D1), Timo Schulte1,2, \\ Oliver Groene (iD) \\ ${ }^{1}$ Chair of Management and Innovation in Health Care, Witten/Herdecke University, Witten, 58448, Germany \\ 2OptiMedis AG, Hamburg, 20095, Germany
}

V1 First published: 01 Dec 2021, 10:1223

https://doi.org/10.12688/f1000research.73875.1

Latest published: 06 Apr 2022, 10:1223

https://doi.org/10.12688/f1000research.73875.2

\section{Abstract}

Background: Hospitalizations of nursing home residents are associated with various health risks. Previous research indicates that, to some extent, hospitalizations of this vulnerable population may be inappropriate and even avoidable. This study aimed to develop a consensus list of hospital discharge diagnoses considered to be nursing home-sensitive, i.e., avoidable.

Methods: The study combined analyses of routine data from six statutory health insurance companies in Germany and a two-stage Delphi panel, enhanced by expert workshop discussions, to identify and corroborate relevant diagnoses. Experts from four different disciplines estimated the proportion of hospitalizations that could potentially have been prevented under optimal conditions.

Results: We analyzed frequencies and costs of data for hospital admissions from 242,236 nursing home residents provided by statutory health insurance companies. We identified 117 hospital discharge diagnoses, which had a frequency of at least $0.1 \%$. We recruited experts (primary care physicians, hospital specialists, nursing home professionals and researchers) to estimate the proportion of potentially avoidable hospitalizations for the 117 diagnoses deemed avoidable in two Delphi rounds ( $n=107$ in Delphi Round 1 and $n=96$ in Delphi Round 2, effective response rate=91\%). A total of 35 diagnoses with high and consistent estimates of the proportion of potentially avoidable hospitalizations were identified as nursing home-sensitive. In an expert workshop $(n=16)$, a further 25 diagnoses were discussed that had not reached the criteria, of which another 23 were consented to be nursing home-sensitive conditions. Extrapolating the frequency and mean costs of these 58 diagnoses to

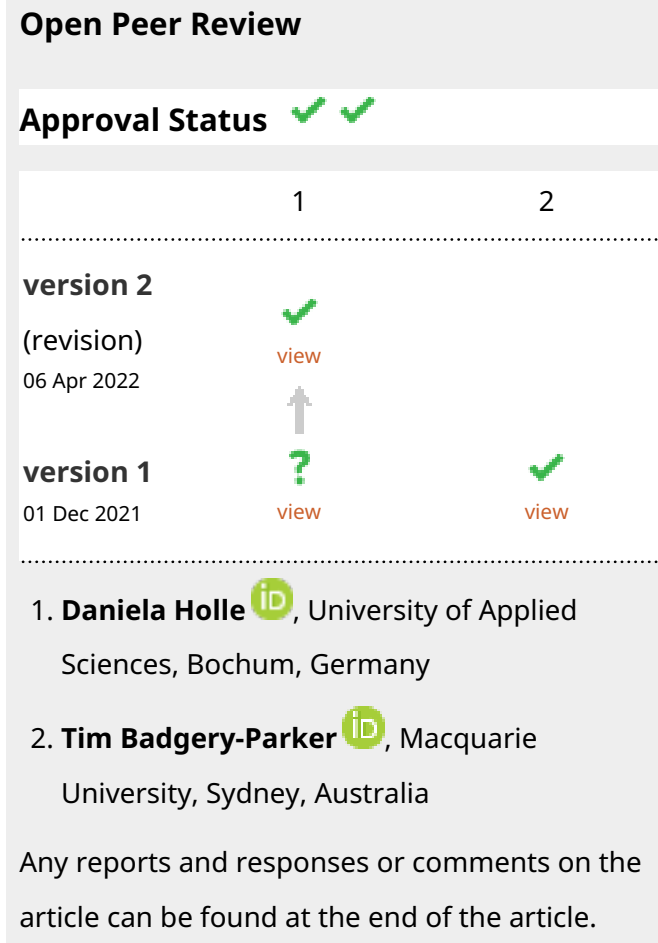


the national German context yielded total potentially avoidable care costs of $€ 768,304,547$, associated with 219,955 nursing homesensitive hospital admissions.

Conclusion: A total of 58 nursing home-relevant diagnoses (ICD-10GM three-digit level) were classified as nursing home-sensitive using an adapted Delphi procedure. Interventions should be developed to avoid hospital admission from nursing homes for these diagnoses.

\section{Keywords}

geriatrics, health services research, potentially avoidable hospitalization, hospitalization, long-term care, nursing homes, nursing home-sensitive conditions

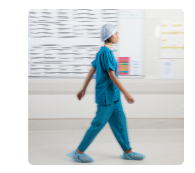

This article is included in the Health Services

gateway.

Corresponding author: Sabine Bohnet-Joschko (sabine.bohnet-joschko@uni-wh.de)

Author roles: Bohnet-Joschko S: Conceptualization, Funding Acquisition, Methodology, Project Administration, Supervision, Validation, Writing - Original Draft Preparation, Writing - Review \& Editing; Valk-Draad MP: Conceptualization, Data Curation, Formal Analysis, Investigation, Methodology, Project Administration, Visualization, Writing - Original Draft Preparation, Writing - Review \& Editing; Schulte T: Conceptualization, Data Curation, Formal Analysis, Funding Acquisition, Investigation, Methodology, Visualization; Groene 0: Conceptualization, Funding Acquisition, Methodology, Supervision, Validation, Writing - Original Draft Preparation, Writing - Review \& Editing

Competing interests: No competing interests were disclosed.

Grant information: This work was supported by the Innovation Committee of the Federal Joint Committee (G-BA) under grant agreement number 01VSF18026.

The funders had no role in study design, data collection and analysis, decision to publish, or preparation of the manuscript.

Copyright: ( $) 2021$ Bohnet-Joschko S et al. This is an open access article distributed under the terms of the Creative Commons Attribution License, which permits unrestricted use, distribution, and reproduction in any medium, provided the original work is properly cited.

How to cite this article: Bohnet-Joschko S, Valk-Draad MP, Schulte T and Groene O. Nursing home-sensitive conditions: analysis of routine health insurance data and modified Delphi analysis of potentially avoidable hospitalizations [version 1; peer review: 1 approved, 1 approved with reservations] F1000Research 2021, 10:1223 https://doi.org/10.12688/f1000research.73875.1

First published: 01 Dec 2021, 10:1223 https://doi.org/10.12688/f1000research.73875.1 


\section{Introduction}

Hospitalizations pose various risks to nursing home residents. Residents may experience reduced functioning upon their return to the nursing home (post-hospital syndrome). ${ }^{1}$ Hospital-acquired conditions may occur, for example, when specific hospital pathogens lead to infections. ${ }^{2}$ Adverse drug effects can also develop, such as medication overdose or administration of the wrong medication. ${ }^{3}$ Cognitively impaired patients often experience loss of orientation as well as confusion in an unfamiliar hospital setting. ${ }^{4}$ Therefore, there should be intense efforts to avoid unnecessary hospitalizations amongst nursing home residents in health systems across the globe.

The issue of preventing hospital admissions has been intensely discussed regarding ambulatory care-sensitive conditions, for which specific indicator sets have been developed to measure the extent and preventability. ${ }^{5-13}$ Ambulatory care (or primary care)-sensitive conditions are those which by expert consensus should not require a hospital admission in the presence of effective primary care. Conditions frequently referred to in existing indicator sets include asthma, chronic obstructive pulmonary disease, congestive heart failure, diabetes mellitus and hypertension, among others. ${ }^{14}$ Indicator sets to measure ambulatory care-sensitive conditions received substantial attention in policy, research, and clinical practice. ${ }^{15}$ Such indicator sets may identify variations in regional hospital admission rates, which can lead to investigations about appropriate care structures, as well as interventions to reduce unnecessary hospital admissions.

Whether ambulatory care-sensitive indicator sets can be applied to hospital admissions from nursing homes has been subject to debate. One argument against the use of ambulatory care-sensitive indicator sets is that nursing home populations differ significantly from the general population. The age structure of nursing home residents, the number of comorbidities, the geriatric disease spectrum, as well as the healing process of the elderly population and typical medical interventions, differ from those of community-dwelling residents. ${ }^{10,12}$ Moreover, the care setting in long-term care facilities, where care is provided by trained nurses and allied professions 24 hours a day, contrasts with community or outpatient care. The characteristics of the nursing home resident population as well as the long-term care setting influence the type of diagnosis that may require hospital admission, its frequency, and its preventability. Therefore, as existing indicators regarding ambulatory care-sensitive conditions are unlikely to be applicable to long-term care settings or patient populations who are nursing home residents, others have urged the need to develop additional nursing homesensitive indicator sets. ${ }^{16}$

Various studies have investigated potentially preventable hospital admissions from the long-term care situations/nursing home setting. ${ }^{8,16-19}$ Earlier studies conducted medical chart reviews and convened panels to gauge the preventability of a hospital admission. Using such an approach, Ouslander et al. ${ }^{8}$ estimated that $67 \%$ of hospital admissions in the USA were preventable. Others, such as Walker et al. ${ }^{17}$ in Canada, adopted existing indicator sets for ambulatory care-sensitive hospital admissions, and used administrative databases to calculate that $55 \%$ of hospital admissions were preventable. In Germany, Leutgeb et al. ${ }^{10}$ compared ambulatory care-sensitive hospital admission rates amongst nursing home and community-dwelling residents, and found that admission rates were significantly higher amongst nursing home residents. Allers et al. cautioned in their systematic review that interventions to reduce hospitalization of nursing home residents should be tailored to health care systems: for policy and clinical practice, it is critical that indicator sets are based on consensus of experts working in the field, that they reflect the characteristic of the national/regional nursing home population and settings, and that they take into account the available health system resources, such as the nursing skills available in the facility, or access to family doctor and specialist visits to the nursing home. ${ }^{20}$

In order to inform policy debate and practical improvement actions to reduce hospital admissions from nursing homes in Germany, this research project aimed to address the following questions: 1 . How often are nursing home residents treated in hospital and what are the main diagnoses and associated costs of these hospital admissions? 2. Which hospital cases are nursing home-sensitive, i.e., at least partially preventable under optimal conditions? 3 . What is the impact of the estimated preventability, in case these optimal care conditions existed, at the national level?

\section{Methods}

Overall approach

For this study, a quantitative mixed-method approach was used, in three consecutive phases. First, an analysis was conducted based on health insurance claims data to identify frequent diagnoses amongst nursing home residents admitted to a hospital. The recommendations of the Working Group for the Survey and Utilization of Secondary Data for the analysis of German health insurance claims data were considered. ${ }^{21}$ These include data quality issues and recommendations on contractual details between researchers and data owners, among others. Second, a RAND/UCLA Appropriateness Method ${ }^{22}$ was executed, in which a Delphi expert panel and expert workshop were combined, to reach expert consensus regarding the extent to which hospital admissions might be prevented. A randomized clinical trial was not appropriate for our nursing home resident population: the decision to hospitalize or not, often influences the patient's 
survival itself in this vulnerable population. Our method yielded the best available scientific evidence with the collective judgment of experts regarding the appropriateness of hospitalization in nursing home residents. And third, an analysis of routine health insurance data was extrapolated to the total German nursing home resident population, based on which the expenses associated with potentially preventable hospitalizations were estimated. The quantitative data analyses were performed with Microsoft Excel 2016 and IBM SPSS Statistics 26.

\section{Ethical considerations}

An ethical approval was not required and therefore waived. We relied on secondary use of anonymous, aggregated routine data and expert health professionals' assessments. We did not conduct human research, interventional and noninterventional clinical studies nor clinical trials. We did not collect nor use data in the form of direct health care data, nor did we use (residual) human material for scientific purposes.

All experts provided their written informed consent to participate voluntarily. The Delphi procedure was conducted pseudonymously: towards the end of the data collection, experts were requested to provide their name and email address in a separate database, so that they could be reached for subsequent Delphi rounds and payment of the incentive. Neither the name nor e-mail address given could be linked to the data collection, although the names of the participants were known to the research team. Nonetheless, the assessments towards the potential of preventability were completely anonymized and uninfluenced by the research team. The voluntary nature and pseudonymity of participation was pointed out, together with complete information on the EU General Data Protection Regulation.

\section{Analysis of routine health insurance data}

We obtained claims data from six statutory health insurance companies, which together provide a representative data set of about $29.6 \%$ of all nursing home residents in the German population. A data request was agreed between the researchers and health insurance companies, whereupon aggregated data on hospital discharge diagnoses were provided. Included were insured persons living in a nursing home in the calendar year 2017, with a hospital discharge diagnosis (coded as ICD-10-GM, three digits) that occurred in more than $0.1 \%$ of this population. Nursing home residents whose insurance period ended in 2017 due to a change in health insurance fund, or who did not have a valid insurance period were excluded from the analysis, to be able to consider insurance utilization without gaps. Insured persons who provided implausible information were also excluded from further analysis. Deceased insured persons, however, were not excluded, because otherwise serious illnesses associated with death in hospital might have been underrepresented, and because nursing home patients have a higher risk of death in hospital than comparable populations. ${ }^{23}$ For the purposes of this study, nursing home residents are those insured persons for whom a start date prior to Jan 1, 2017, was documented for both a need for long-term care and for full inpatient care in an approved nursing home, pursuant to Section 43 of the German Social Code, Book XI, and who - except for deceased insured persons - consistently had these care services in 2017.

The hospital discharge diagnoses had to have a discharge date within the calendar year 2017. This excluded cases who were admitted to hospital in 2017 or before, but discharged in a later calendar year, which was considered unproblematic as, in contrast, cases were included that were admitted before 2017 but discharged in 2017. In addition, the average costs per hospital case were evaluated. Here, the average total amount paid by social health insurance for the case was used, not just the cost share that would result from the diagnosis-related group (DRG) of the principal diagnosis. The total amount was evaluated entirely on the discharge date. The list of principal hospital diagnoses was aggregated from all insurance companies and sorted in descending order for the analysis, to determine ICD-10 diagnoses with highest frequency and cost. The exact data request is included in our Extended data for this publication (see Data availability section).

\section{Delphi study and expert workshop}

We conducted the modified Delphi study between December 2019 and July 2020 as a two-round online tool followed by an expert workshop in September 2020, combining the strengths of the anonymous questioning of experts with the deeper insight emerging from the discussions at the moderated workshop. The online questionnaire and the expert workshop background information and workshop task are included in the Extended data.

In the online tool, experts of four different disciplines (physicians working in outpatient and inpatient care, nursing professionals, and researchers) were asked to estimate the proportion of potentially avoidable hospitalizations identified in the health insurance claims data. The assessment of preventability was under the assumptions of optimal, but still realistic conditions: access to trained personnel, resources and infrastructure for monitoring and nursing, and cooperation agreements with ambulatory general and specialist care providers where needed. For each discharge diagnosis, a short description and a link to the official definition on the German ICD-10 classification were added, to facilitate the assessment for all experts. ICD-10 codes were restricted to the three-digit level to ensure comprehension and usability of 
the list of diagnoses by all professions. The differentiation for subgroups of the ICD-10 three-digit code was indicated by experts via the numerical estimation of the potential preventability. The experts made their assessments on a scale from $0 \%$ to $100 \%$ in 5\% increments ( $0 \%$ meaning hospitalization was unavoidable, $100 \%$ meaning all patients could have been treated in the nursing home). In addition, Delphi participants were provided the opportunity to offer voluntary comments for each ICD-10 code, which became mandatory in the event that experts were unable to give a quantitative estimate of the preventability. LimeSurvey (Version 3.27.20+211012) was selected as the tool for online data collection and was customized specifically for this study. Feedback was obtained and processed from four experts regarding the questionnaire tool, to improve overall presentation and instructions for the experts as well as the presentation of the list of ICD-10 codes. Comprehension and practicability of the online evaluation were tested in a pilot group, technical errors were corrected and ambiguities in the content were clarified.

To recruit the experts, we used the Delphi funnel, a panel management model, according to Donohoe et al. ${ }^{24}$ Funneling constituted in first identifying and selecting potential experts or expert groups, after which we approached them. Additionally, gatekeepers were identified to help pinpoint those individuals who would have knowledge of the topic under study. ${ }^{25}$ The professional networks of the research team were consulted, and potentially eligible experts were contacted through posts on websites and social media such as LinkedIn, via newsletter announcements, through personal email as well as email distribution lists, on personal recommendation, by phone, and through personal visits.

A Delphi group size depends on group dynamics for arriving a consensus among experts. ${ }^{26}$ It is also subject to the expected loss-to follow up because of attrition. ${ }^{24}$ The literature recommends 10 to $18^{26}$ or, in case of high attrition, up to 90 experts $^{24}$ on a Delphi panel. The Delphi panel should be large enough to reach a sufficient number of perspectives from the "inside". ${ }^{26}$ A detailed expert selection criteria list was developed, as purposeful panelist selection can reduce attrition due to loss of interest or frustration. ${ }^{24}$ For our study, experts should either have practical experience from the sectors involved in the treatment of nursing home residents, play an important role in the decision about hospital treatment, or have published scientific research related to the care of elderly patients. According to these recommendations and in order to ensure a large, methodologically robust and balanced sample of experts for the consensus ratings, we chose to reach out to heterogeneous experts from four different disciplines and planned to recruit 100 experts: 30 outpatient/clinical physicians each, 30 nursing professionals and 10 scientists.

Second, we supported the participants' engagement by distributing an introductory package along with the invitation to participate in the assessment. ${ }^{24}$ The preparation of the experts was important in order not to compromise the response rate in future rounds. ${ }^{25}$ Therefore, before the first Delphi round, experts were informed about the content, the objectives of the research project, what they would be asked to do, how much time they would be expected to contribute, what use would be made of the information they provided, the voluntary nature of participation, and data confidentiality. ${ }^{25}$ Additional information was also given to pseudonymity of the study (see the Ethical considerations section). After giving their informed consent, experts were invited to participate in this study.

Third, results of the first round should be distributed to the panel. ${ }^{24}$ Therefore, the data collection tool for the second round integrated the results of the expert ratings from the first round. The RAND/UCLA-Appropriateness Method ${ }^{22}$ is visualized in Figure 1. Expert panel management for the Delphi process was integrated as shown in the Delphi funnel. ${ }^{24}$

To increase the willingness to participate, a compensation of $100 €$ was offered for successful participation in both rounds of the survey. For each of the Delphi rounds, the acquisition of experts in that group was discontinued as soon as the required number of participants in each group was reached.

The data from the first Delphi round were analyzed as follows: the median and its interquartile range (containing 50\% of all assessments around the median) was reported for each ICD-10-GM three-digit code. Based on this information, the participants were asked to quantify the proportion of potentially avoidable hospitalization again in the second round. If the estimation of the proportion either fell outside the given interquartile range or could not be estimated, the comment field was mandatory again; otherwise, the participants could include extra comments voluntarily. Comments would reveal information about the reasons behind a deviating answer, possible disruptors or problematic conditions complicating the avoidance of hospitalization. Only the comments from the first and second rounds of those ICD-10 codes reaching relevant but dispersed preventability estimates after both online Delphi rounds (conditions described in workshop section) were looked at. This was solely done to be able to identify possible difficulties in the estimation of preventability to be discussed at the expert workshop. Content analysis on this subpart of comments identified themes, which were grouped and described close to the original text, to stimulate the subsequent discussion in the expert workshop. ${ }^{27}$ We followed SRQR guidelines for this small qualitative component of our research: the analysis of a subpart of free text comments collected as part of our Delphi study. However, this only corresponds to a fraction of our research, 


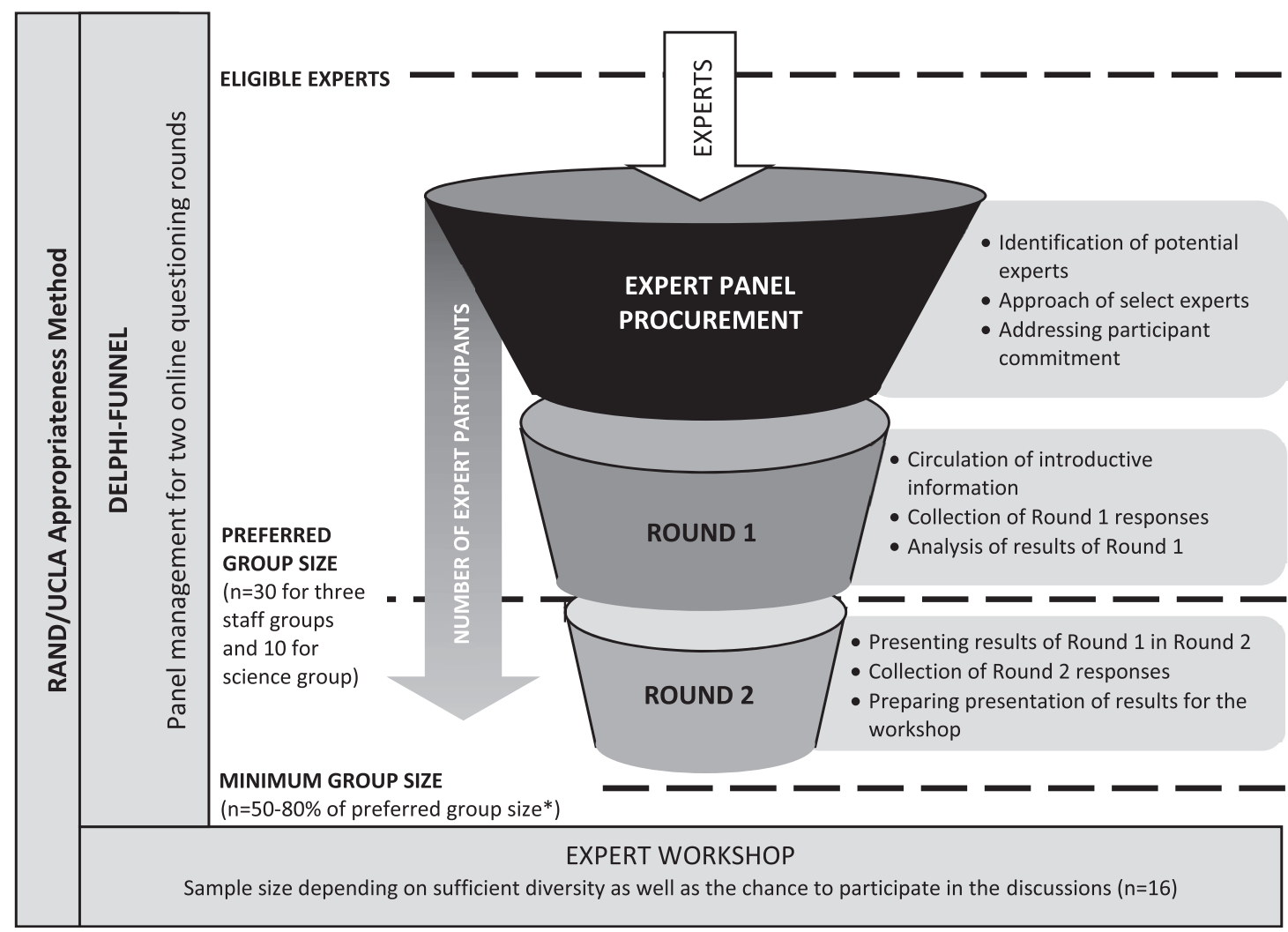

Figure 1. RAND/UCLA-appropriateness method in the nursing home-sensitive conditions study: Delphi expert panel management combined with expert workshop.* *Source: Delphi funnel ${ }^{24}$; minimum group size. ${ }^{32-35}$

the much larger part of our study being based on quantitative consensus techniques and the analysis of administrative health insurance claims data. These were analyzed and reported according to STROBE.

A multidisciplinary expert workshop was convened to discuss those diagnoses, for which the Delphi panel generated highly dispersed data. For face-to-face discussions, it is recommended to have a panel size that permits sufficient diversity, while ensuring that all have a chance to participate. ${ }^{22}$ This panel consisted of sixteen experts. Again, all four disciplines were represented in equal proportions. A total of 10 out of 16 experts had taken part in the online Delphi rounds. The remaining six experts were provided with the same information on the project from the online rounds. All experts received the same information about the goal and content of the workshop, and all gave their informed consent to take part in course of registration for the online workshop. ICD-10 codes with an assessed preventability potential (median $\geq 75 \%$ ) and a narrow range of dispersion (dispersion around median $\leq 15 \%$ ), were considered directly eligible for the list of nursing home-sensitive conditions. In case the preventability potential of at least $75 \%$ was not comprised in the range of $75 \%$ of all expert assessments, the ICD-10 codes were excluded. For all other ICD-10 codes, statistical data (median, modal values, dispersion parameters, bar charts) and categorized comments from the Delphi questionnaire were provided for the expert workshop. The ICD-10 codes were thematically clustered where possible and distributed across three working groups, while the experts were assigned to the working groups according to their expertise.

\section{Comparing nursing home-sensitive with ambulatory care-sensitive conditions}

After preventability estimates were corroborated, the composition and the preventability estimates of these conditions were compared to the results of Sundmacher $\mathrm{t} \mathrm{al}^{7}$ They developed a list of ambulatory care-sensitive conditions for the outpatient-German setting. They sorted 258 ICD-10 conditions, expected to be ambulatory care-sensitive by experts, into 40 groups according to disease categories. Each group comprised three- and four-digit ICD-10, because, in some ICD-10, potential preventability was attributed to subcategories only. After that, the potential preventability for each of these groups was estimated. The estimates of preventability for these groups of ICD-10 ranged between 55-96\%. ${ }^{7}$ Of these groups, 22 had an estimated preventability of more than $85 \%$ and were posed as core ambulatory care-sensitive conditions. ${ }^{7}$ The more common ICD-10 discharge diagnoses among nursing home residents, i.e., with a frequency of 
at least $0.1 \%$, as well as the nursing home-sensitive conditions we found in our study, were then compared to both groups: the ambulatory care-sensitive conditions and the core ambulatory care-sensitive conditions.

\section{Extrapolating the costs of potentially avoidable hospitalizations}

Following the expert consensus process, based on which consensus on a list of nursing home-sensitive hospital admissions was obtained, we used health insurance claims data to calculate the total amount of costs associated with preventable hospital admissions from nursing homes in Germany. We used data from six health insurance companies to assess the total costs associated with the hospitalization case for each diagnosis, and data from the Federal Statistical Office to identify the total number of people living in long-term care facilities in Germany. ${ }^{28}$ We calculated the number of hospital cases and multiplied those with the average case costs for each diagnosis, which resulted in the total costs of cases with the respective discharge diagnoses. This, further multiplied by the proportion of potential preventability then resulted in the total amount of health care system costs that could potentially be avoided (given optimal care conditions).

\section{Results}

Analysis of routine health insurance data

We received data from six German statutory health insurance companies according to our data request, covering information on 242,236 nursing home residents. Where data was provided in deviating format, we cleaned and prepared the datasets so they could be aggregated into a global data set. Data included the ICD-10 code, the proportion of hospital admission from nursing-home/long-term care, the gender and age distribution (categorized in 5-year strata) and the mean total cost per case. The age and gender distributions are presented in Table 1 and the gender-differentiated number of hospitalizations, hospitalization proportions and ratios are presented in Table 2. The data received from six health insurance companies were merged and the results are presented in Table 3. This table shows all the hospital discharge diagnoses with a frequency of at least $0.1 \%$ in our sample, sorted according to their frequency in descending order, together with a short description of the ICD-10 code, case counts, percentage as well as cumulative percentage of hospital discharge diagnoses from long-term care, and average cost per case for each ICD-10 code.

Over $85 \%$ of nursing home residents in our sample were at least 65 years old (206,503 persons of the total sample; Table 1). In the aggregated data set, $44 \%$ of fully insured nursing home residents were hospitalized. Thus in 2017 , there were 79 hospital admissions for every 100 nursing home residents (Table 2). The percentage of persons with one or more hospitalizations was slightly higher among men (48\%) than among women (44\%; Table 2).

Table 1. Age and gender distribution of merged health insurance company data of nursing home residents.

\begin{tabular}{|c|c|c|c|c|c|c|}
\hline $\begin{array}{l}\text { Age group } \\
\text { (years of age) }\end{array}$ & $\begin{array}{l}\text { Male } \\
\text { (N) }\end{array}$ & $\begin{array}{l}\text { Female } \\
\text { (N) }\end{array}$ & $\begin{array}{l}\text { Total } \\
\text { (N) }\end{array}$ & $\begin{array}{l}\text { Male } \\
(\%)\end{array}$ & $\begin{array}{l}\text { Female } \\
(\%)\end{array}$ & $\begin{array}{l}\text { Total } \\
(\%)\end{array}$ \\
\hline $0-14$ & 215 & 323 & 538 & $0.09 \%$ & $0.13 \%$ & $0.22 \%$ \\
\hline $15-19$ & 459 & 291 & 750 & $0.19 \%$ & $0.12 \%$ & $0.31 \%$ \\
\hline $20-24$ & 625 & 538 & 1,163 & $0.26 \%$ & $0.22 \%$ & $0.48 \%$ \\
\hline $25-29$ & 1,035 & 916 & 1,951 & $0.43 \%$ & $0.38 \%$ & $0.81 \%$ \\
\hline $30-34$ & 1,276 & 949 & 2,225 & $0.53 \%$ & $0.39 \%$ & $0.92 \%$ \\
\hline $35-39$ & 1,257 & 996 & 2,253 & $0.52 \%$ & $0.41 \%$ & $0.93 \%$ \\
\hline $40-44$ & 1,376 & 1,019 & 2,395 & $0.57 \%$ & $0.42 \%$ & $0.99 \%$ \\
\hline $45-49$ & 1,921 & 1,711 & 3,632 & $0.79 \%$ & $0.71 \%$ & $1.50 \%$ \\
\hline $50-54$ & 3,112 & 2,658 & 5,770 & $1.28 \%$ & $1.10 \%$ & $2.38 \%$ \\
\hline $55-59$ & 3,903 & 3,292 & 7,195 & $1.61 \%$ & $1.36 \%$ & $2.97 \%$ \\
\hline $60-64$ & 4,298 & 3,563 & 7,861 & $1.77 \%$ & $1.47 \%$ & $3.25 \%$ \\
\hline $65-69$ & 5,011 & 4,540 & 9,551 & $2.07 \%$ & $1.87 \%$ & $3.94 \%$ \\
\hline $70-74$ & 5,271 & 6,638 & 11,909 & $2.18 \%$ & $2.74 \%$ & $4.92 \%$ \\
\hline $75-79$ & 9,048 & 16,556 & 25,604 & $3.74 \%$ & $6.83 \%$ & $10.57 \%$ \\
\hline $80-84$ & 10,454 & 29,364 & 39,818 & $4.32 \%$ & $12.12 \%$ & $16.44 \%$ \\
\hline $85-89$ & 9,917 & 40,872 & 50,789 & $4.09 \%$ & $16.87 \%$ & $20.97 \%$ \\
\hline$\geq 90$ & 8,140 & 60,692 & 68,832 & $3.36 \%$ & $25.05 \%$ & $28.42 \%$ \\
\hline Total & 67,317 & 174,919 & 242,236 & $27.79 \%$ & $72.21 \%$ & $100.00 \%$ \\
\hline
\end{tabular}


Table 2. Gender-differentiated numbers of hospitalizations, hospitalization proportions and ratios.

\begin{tabular}{|c|c|c|c|c|c|c|}
\hline & $\begin{array}{l}\text { Number of } \\
\text { nursing } \\
\text { home } \\
\text { residents } \\
\left(\mathrm{NHR}^{*}\right)(\mathrm{A})\end{array}$ & $\begin{array}{l}\text { Proportion } \\
\text { of NHR on } \\
\text { total } \\
\text { sample } \\
(\mathrm{A} / 242,236)\end{array}$ & $\begin{array}{l}\text { Number of } \\
\text { hospitalized } \\
\text { NHR (B) }\end{array}$ & $\begin{array}{l}\text { Total number of } \\
\text { hospitalizations } \\
\text { (C) }\end{array}$ & $\begin{array}{l}\text { Proportion } \\
\text { of hospital } \\
\text { cases in } \\
\text { total } \\
\text { sample } \\
\text { (B/A) }\end{array}$ & $\begin{array}{l}\text { Ratio of total } \\
\text { number of } \\
\text { hospitalizations } \\
\text { to total number } \\
\text { of hospitalized } \\
\text { NHR (C/B) }\end{array}$ \\
\hline Male & 67,317 & $28 \%$ & 32,275 & 62,302 & $48 \%$ & 1,93 \\
\hline Female & 174,919 & $72 \%$ & 74,679 & 124,269 & $43 \%$ & 1,66 \\
\hline Total & 242,236 & $100 \%$ & 106,954 & 191,174 & $44 \%$ & 1,79 \\
\hline
\end{tabular}

*NHR: nursing home residents.

In total, the top 25 most common discharge diagnoses accounted for 97,378 cases (Table 3). They covered about half of all hospital cases. About one-third of all hospital cases accounted for one of the following diagnoses: heart failure, pneumonia, fracture of the femur, dehydration, diseases of the urinary tract, intracranial injuries, sepsis, cerebral infarction, and epilepsy. Diseases of the central and peripheral nervous system most frequently led to inpatient treatment of nursing home residents (18\%), closely followed by diseases of the respiratory tract (17\%) and the digestive tract (15\%). The distribution of treatment costs showed a partly different ranking. Neurological diseases such as stroke and epilepsy accounted for the largest share of costs $(21 \%)$, followed by diseases of the musculoskeletal system (17\%), the respiratory system (16\%), and the cardiovascular system (13\%). From a macroeconomic perspective, it is particularly interesting to note that in the individual organ system groups, a particularly high proportion of costs could be allocated to a few discharge diagnoses. These included fractures in musculoskeletal diseases (over 80\%) and pneumonia in respiratory diseases (almost 60\%).

The average cost of a nursing home resident hospital case in 2017 in the sample was $€ 4,030$ (191,174 hospitalizations with hospital costs of $€ 770,368,090$; Tables 3 and 7). Extending Table 3 to the defined cut-off point of $0.1 \%$ share of all hospital cases, 117 different ICD-10 diagnoses were considered for the Delphi study (totaling 157,322 cases).

\section{Delphi study and expert workshop}

We were able to exceed our recruitment goal of $n=100$ experts for the Delphi study, as defined in the protocol, to a number of 107. Of the 107 experts participating in the first round of the Delphi study, 104 (97.2\%) had indicated their name and e-mail address and were invited to the second round. Of these, $96(92.3 \%)$ followed the invitation (Table 4) and 95 completed the second round successfully so that responses were usable (effective response rate 91\%). There were no significant differences in age, gender, and years of experience between responders and non-responders.

Details of the experts' estimate of the preventability of hospitalizations from the nursing home following the Delphi rounds and the expert workshop are reported in Table 5. In the first Delphi round, experts estimated the proportion of potentially avoidable hospitalizations for 117 ICD-10 codes which were identified in the previous step, based on the analysis of routine health insurance data. Experts were asked to provide their estimations assuming optimal structural and care conditions. Where ICD-10 codes were not assessed by six or more experts, we reviewed the comments to identify why experts had difficulties in assessing the potential preventability of individual ICD-10 codes. This concerned 20 of the 117 ICD-10 codes (17\%). To avoid further assessment difficulties, notes were included in the explanations for these ICD-10 codes, or their lay-out was changed for the second Delphi round. Furthermore, ICD-10 codes were planned to be excluded when at least $75 \%$ of the experts estimated the preventability as zero in the first round. This condition was never met. Secondly, ICD-10 codes were planned to be combined if the diseases were very similar (in terms of symptoms, diagnosis, prognosis, treatment) and the proportions of potential preventability were nearly identical. Two conditions for "nearly identical potential preventability" had to be fulfilled: the medians of the estimated preventability should not differ by more than $5 \%$ and the limits of the interquartile range had to be less than $10 \%$ apart). Neither conditions were met sufficiently. Therefore, for the second round, all 117 hospital discharge diagnoses were assessed again.

Comparing the responses to the Delphi rounds, we found no differences in the median estimates of the preventability of the 117 ICD-10 codes; however, the scatter range decreased significantly. The width of the interquartile range decreased, for all ICD-10 codes together, from $42.3 \%$ to $5.5 \%$ on average. Comparing the respective participants' assessments in both online questionnaire Delphi rounds, both assessments were very close together: the median difference between round 1 and 2 was maximum 5\% for 114 of the 117 ICD-10 codes, and maximum 10\% for the three remaining ICD-10 codes. 
Table 3. Merged results of analyses on data received from six health insurance companies on nursing home hospitalizations in Germany.*

\begin{tabular}{|c|c|c|c|c|c|c|}
\hline No. & $\begin{array}{l}\text { ICD-10- GM } \\
\text { (three digits) }\end{array}$ & ICD-name & Cases & $\begin{array}{l}\% \\
\text { hospital } \\
\text { cases }\end{array}$ & $\begin{array}{l}\text { \% } \\
\text { hospital } \\
\text { cases } \\
\text { (cumulated) }\end{array}$ & $\begin{array}{l}\text { Mean } \\
\text { cost per } \\
\text { hospital } \\
\text { case }\end{array}$ \\
\hline 1 & I50 & Heart failure & 9,924 & $5.19 \%$ & $5.19 \%$ & $3,683 €$ \\
\hline 2 & J18 & $\begin{array}{l}\text { Pneumonia (pathogen not } \\
\text { specified) }\end{array}$ & 8,503 & $4.45 \%$ & $9.64 \%$ & $3,671 €$ \\
\hline 3 & $\mathrm{~S} 72$ & Fracture of the femur & 8,029 & $4.20 \%$ & $13.84 \%$ & $7,794 €$ \\
\hline 4 & E86 & Lack of volume & 6,579 & $3.44 \%$ & $17.28 \%$ & $2,549 €$ \\
\hline 5 & N39 & $\begin{array}{l}\text { Other diseases of the urinary } \\
\text { system }\end{array}$ & 6,150 & $3.22 \%$ & $20.50 \%$ & $2,613 €$ \\
\hline 6 & S06 & Intracranial injury & 5,054 & $2.64 \%$ & $23.14 \%$ & $2,104 €$ \\
\hline 7 & A41 & Other sepsis & 4,508 & $2.36 \%$ & $25.50 \%$ & $4,984 €$ \\
\hline 8 & J69 & $\begin{array}{l}\text { Pneumonia due to solid and } \\
\text { liquid substance }\end{array}$ & 4,486 & $2.35 \%$ & $27.85 \%$ & $4,007 €$ \\
\hline 9 & I63 & Cerebral infarction & 4,410 & $2.31 \%$ & $30.15 \%$ & $6,228 €$ \\
\hline 10 & G40 & Epilepsy & 4,149 & $2.17 \%$ & $32.32 \%$ & $3,371 €$ \\
\hline 11 & J44 & $\begin{array}{l}\text { Other chronic obstructive } \\
\text { pulmonary disease }\end{array}$ & 4,125 & $2.16 \%$ & $34.48 \%$ & $4,346 €$ \\
\hline 12 & E11 & Diabetes mellitus, type 2 & 3,173 & $1.66 \%$ & $36.14 \%$ & $4,197 €$ \\
\hline 13 & K56 & $\begin{array}{l}\text { Paralytic ileus and intestinal } \\
\text { obstruction without hernia }\end{array}$ & 2,913 & $1.52 \%$ & $37.66 \%$ & $4,418 €$ \\
\hline 14 & $J 20$ & Acute bronchitis & 2,563 & $1.34 \%$ & $39.00 \%$ & $2,692 €$ \\
\hline 15 & $\mathrm{I} 70$ & Atherosclerosis & 2,487 & $1.30 \%$ & $40.31 \%$ & $6,912 €$ \\
\hline 16 & K92 & $\begin{array}{l}\text { Other diseases of the digestive } \\
\text { system }\end{array}$ & 2,396 & $1.25 \%$ & $41.56 \%$ & $2,277 €$ \\
\hline 17 & SOO & Superficial injury of the head & 2,362 & $1.24 \%$ & $42.79 \%$ & $1,122 €$ \\
\hline 18 & N17 & Acute renal failure & 2,328 & $1.22 \%$ & $44.01 \%$ & $4,375 €$ \\
\hline 19 & S32 & $\begin{array}{l}\text { Fracture of the lumbar spine } \\
\text { and pelvis }\end{array}$ & 2,162 & $1.13 \%$ & $45.14 \%$ & $4,166 €$ \\
\hline 20 & F05 & $\begin{array}{l}\text { Delirium not caused by alcohol } \\
\text { or other psychotropic } \\
\text { substance }\end{array}$ & 2,039 & $1.07 \%$ & $46.21 \%$ & $5,915 €$ \\
\hline 21 & J15 & $\begin{array}{l}\text { Pneumonia due to bacteria, } \\
\text { not classified elsewhere }\end{array}$ & 2,012 & $1.05 \%$ & $47.26 \%$ & $4,723 €$ \\
\hline 22 & N30 & Cystitis & 1,833 & $0.96 \%$ & $48.22 \%$ & $2,771 €$ \\
\hline 23 & A09 & $\begin{array}{l}\text { Other and unspecified } \\
\text { gastroenteritis and colitis of } \\
\text { infections and unspecified } \\
\text { origin }\end{array}$ & 1,782 & $0.93 \%$ & $49.15 \%$ & $2,210 €$ \\
\hline 24 & R55 & Syncope and collapse & 1,720 & $0.90 \%$ & $50.05 \%$ & $1,933 €$ \\
\hline 25 & $\mathrm{~F} 20$ & Schizophrenia & 1,691 & $0.88 \%$ & $50.94 \%$ & $8,144 €$ \\
\hline 26 & $\mathrm{I} 10$ & $\begin{array}{l}\text { Essential (primary) } \\
\text { hypertension }\end{array}$ & 1,673 & $0.88 \%$ & $51.81 \%$ & $2,069 €$ \\
\hline 27 & I21 & Acute myocardial infarction & 1,612 & $0.84 \%$ & $52.66 \%$ & $5,000 €$ \\
\hline 28 & K80 & Cholelithiasis & 1,595 & $0.83 \%$ & $53.49 \%$ & $4,847 €$ \\
\hline 29 & K59 & $\begin{array}{l}\text { Other functional intestinal } \\
\text { disorders }\end{array}$ & 1,586 & $0.83 \%$ & $54.32 \%$ & $1,930 €$ \\
\hline 30 & $\mathrm{~S} 42$ & $\begin{array}{l}\text { Fracture of shoulder and } \\
\text { upper arm }\end{array}$ & 1,407 & $0.74 \%$ & $55.06 \%$ & $4,903 €$ \\
\hline
\end{tabular}


Table 3. Continued

\begin{tabular}{|c|c|c|c|c|c|c|}
\hline No. & $\begin{array}{l}\text { ICD-10- GM } \\
\text { (three digits) }\end{array}$ & ICD-name & Cases & $\begin{array}{l}\% \\
\text { hospital } \\
\text { cases }\end{array}$ & $\begin{array}{l}\% \\
\text { hospital } \\
\text { cases } \\
\text { (cumulated) }\end{array}$ & $\begin{array}{l}\text { Mean } \\
\text { cost per } \\
\text { hospital } \\
\text { case }\end{array}$ \\
\hline 31 & $J 22$ & $\begin{array}{l}\text { Unspecified acute lower } \\
\text { respiratory infection }\end{array}$ & 1,400 & $0.73 \%$ & $55.79 \%$ & $2,923 €$ \\
\hline 32 & A46 & Erysipelas & 1,355 & $0.71 \%$ & $56.50 \%$ & $2,894 €$ \\
\hline 33 & G45 & $\begin{array}{l}\text { Transient cerebral ischemic } \\
\text { attacks and related syndromes }\end{array}$ & 1,345 & $0.70 \%$ & $57.20 \%$ & $3,493 €$ \\
\hline 34 & $\mathrm{~K} 21$ & $\begin{array}{l}\text { Gastro-esophageal reflux } \\
\text { disease }\end{array}$ & 1,328 & $0.69 \%$ & $57.89 \%$ & $2,215 €$ \\
\hline 35 & K29 & Gastritis and duodenitis & 1,319 & $0.69 \%$ & $58.58 \%$ & $2,314 €$ \\
\hline 36 & N13 & $\begin{array}{l}\text { Obstructive and reflux } \\
\text { uropathy }\end{array}$ & 1,246 & $0.65 \%$ & $59.24 \%$ & $2,813 €$ \\
\hline 37 & I48 & Atrial fibrillation and flutter & 1,176 & $0.62 \%$ & $59.85 \%$ & $2,959 €$ \\
\hline 38 & D50 & Iron deficiency anemia & 1,159 & $0.61 \%$ & $60.46 \%$ & $3,035 €$ \\
\hline 39 & S22 & $\begin{array}{l}\text { Fracture of rib(s), sternum and } \\
\text { thoracic spine }\end{array}$ & 1,130 & $0.59 \%$ & $61.05 \%$ & $3,263 €$ \\
\hline 40 & $\mathrm{~A} 04$ & $\begin{array}{l}\text { Other bacterial intestinal } \\
\text { infections }\end{array}$ & 1,062 & $0.56 \%$ & $61.60 \%$ & $3,950 €$ \\
\hline 41 & L89 & Pressure ulcer & 1,043 & $0.55 \%$ & $62.15 \%$ & $8,282 €$ \\
\hline 42 & T85 & $\begin{array}{l}\text { Complications of other internal } \\
\text { prosthetic devices, implants } \\
\text { and grafts }\end{array}$ & 986 & $0.52 \%$ & $62.67 \%$ & $3,247 €$ \\
\hline 43 & F06 & $\begin{array}{l}\text { Other mental disorders due to } \\
\text { known physiological condition }\end{array}$ & 969 & $0.51 \%$ & $63.17 \%$ & $6,953 €$ \\
\hline 44 & G20 & Parkinson's disease & 952 & $0.50 \%$ & $63.67 \%$ & $5,167 €$ \\
\hline 45 & $\mathrm{C} 44$ & $\begin{array}{l}\text { Other and unspecified } \\
\text { malignant neoplasm of skin }\end{array}$ & 874 & $0.46 \%$ & $64.13 \%$ & $3,017 €$ \\
\hline 46 & T84 & $\begin{array}{l}\text { Complications of internal } \\
\text { orthopedic prosthetic devices, } \\
\text { implants and grafts }\end{array}$ & 858 & $0.45 \%$ & $64.58 \%$ & $9,410 €$ \\
\hline 47 & E87 & $\begin{array}{l}\text { Other disorders of fluid, } \\
\text { electrolyte and acidbase } \\
\text { balance }\end{array}$ & 857 & $0.45 \%$ & $65.02 \%$ & $2,851 €$ \\
\hline 48 & I80 & $\begin{array}{l}\text { Thrombosis, phlebitis and } \\
\text { thrombophlebitis }\end{array}$ & 847 & $0.44 \%$ & $65.47 \%$ & $2,256 €$ \\
\hline 49 & K57 & $\begin{array}{l}\text { Diverticular disease of } \\
\text { intestine }\end{array}$ & 845 & $0.44 \%$ & $65.91 \%$ & $4,114 €$ \\
\hline 50 & $\mathrm{H} 25$ & Age-related cataract & 830 & $0.43 \%$ & $66.34 \%$ & $1,517 €$ \\
\hline 51 & B99 & $\begin{array}{l}\text { Other and unspecified } \\
\text { infectious diseases }\end{array}$ & 819 & $0.43 \%$ & $66.77 \%$ & $2,632 €$ \\
\hline 52 & G30 & Alzheimer's disease & 815 & $0.43 \%$ & $67.20 \%$ & $5,989 €$ \\
\hline 53 & J96 & $\begin{array}{l}\text { Respiratory failure, not } \\
\text { elsewhere classified }\end{array}$ & 761 & $0.40 \%$ & $67.60 \%$ & $9,046 €$ \\
\hline 54 & S82 & $\begin{array}{l}\text { Fracture of lower leg, including } \\
\text { ankle }\end{array}$ & 753 & $0.39 \%$ & $67.99 \%$ & $4,785 €$ \\
\hline 55 & S52 & Fracture of forearm & 729 & $0.38 \%$ & $68.37 \%$ & $3,218 €$ \\
\hline 56 & I26 & Pulmonary embolism & 714 & $0.37 \%$ & $68.75 \%$ & $3,917 €$ \\
\hline 57 & N18 & Chronic kidney disease (CKD) & 714 & $0.37 \%$ & $69.12 \%$ & $4,542 €$ \\
\hline 58 & $\mathrm{~K} 22$ & Other diseases of esophagus & 707 & $0.37 \%$ & $69.49 \%$ & $3,759 €$ \\
\hline 59 & G41 & Status epilepticus & 677 & $0.35 \%$ & $69.84 \%$ & $5,775 €$ \\
\hline 60 & $\mathrm{~F} 25$ & Schizoaffective disorders & 663 & $0.35 \%$ & $70.19 \%$ & $8,356 €$ \\
\hline
\end{tabular}


Table 3. Continued

\begin{tabular}{|c|c|c|c|c|c|c|}
\hline No. & $\begin{array}{l}\text { ICD-10- GM } \\
\text { (three digits) }\end{array}$ & ICD-name & Cases & $\begin{array}{l}\% \\
\text { hospital } \\
\text { cases }\end{array}$ & $\begin{array}{l}\% \\
\text { hospital } \\
\text { cases } \\
\text { (cumulated) }\end{array}$ & $\begin{array}{l}\text { Mean } \\
\text { cost per } \\
\text { hospital } \\
\text { case }\end{array}$ \\
\hline 61 & S70 & $\begin{array}{l}\text { Superficial injury of hip and } \\
\text { thigh }\end{array}$ & 653 & $0.34 \%$ & $70.53 \%$ & $1,510 €$ \\
\hline 62 & R31 & Hematuria & 650 & $0.34 \%$ & $70.87 \%$ & $2,084 €$ \\
\hline 63 & D64 & Other anemias & 639 & $0.33 \%$ & $71.21 \%$ & $2,810 €$ \\
\hline 64 & $\mathrm{R} 40$ & Somnolence, stupor and coma & 636 & $0.33 \%$ & $71.54 \%$ & $2,220 €$ \\
\hline 65 & F10 & Alcohol related disorders & 614 & $0.32 \%$ & $71.86 \%$ & $3,334 €$ \\
\hline 66 & S30 & $\begin{array}{l}\text { Superficial injury of abdomen, } \\
\text { lower back, pelvis and external } \\
\text { genitals }\end{array}$ & 602 & $0.31 \%$ & $72.17 \%$ & $1,463 €$ \\
\hline 67 & K52 & $\begin{array}{l}\text { Other and unspecified } \\
\text { noninfective gastroenteritis } \\
\text { and colitis }\end{array}$ & 600 & $0.31 \%$ & $72.49 \%$ & $2,600 €$ \\
\hline 68 & T82 & $\begin{array}{l}\text { Complications of cardiac and } \\
\text { vascular prosthetic devices, } \\
\text { implants and grafts }\end{array}$ & 594 & $0.31 \%$ & $72.80 \%$ & $5,690 €$ \\
\hline 69 & T83 & $\begin{array}{l}\text { Complications of } \\
\text { genitourinary prosthetic } \\
\text { devices, implants and grafts }\end{array}$ & 586 & $0.31 \%$ & $73.11 \%$ & $1,898 €$ \\
\hline 70 & M54 & Dorsalgia & 576 & $0.30 \%$ & $73.41 \%$ & $2,519 €$ \\
\hline 71 & I61 & $\begin{array}{l}\text { Nontraumatic intracerebral } \\
\text { hemorrhage }\end{array}$ & 561 & $0.29 \%$ & $73.70 \%$ & $7,477 €$ \\
\hline 72 & C34 & $\begin{array}{l}\text { Malignant neoplasm of } \\
\text { bronchus and lung }\end{array}$ & 552 & $0.29 \%$ & $73.99 \%$ & $4,163 €$ \\
\hline 73 & K55 & Vascular disorders of intestine & 546 & $0.29 \%$ & $74.27 \%$ & $5,838 €$ \\
\hline 74 & R07 & Pain in throat and chest & 542 & $0.28 \%$ & $74.56 \%$ & $1,260 €$ \\
\hline 75 & $\mathrm{R} 13$ & Aphagia and dysphagia & 517 & $0.27 \%$ & $74.83 \%$ & $2,202 €$ \\
\hline 76 & C50 & Malignant neoplasm of breast & 507 & $0.27 \%$ & $75.09 \%$ & $4,532 €$ \\
\hline 77 & F33 & $\begin{array}{l}\text { Major depressive disorder, } \\
\text { recurrent }\end{array}$ & 505 & $0.26 \%$ & $75.36 \%$ & $8,731 €$ \\
\hline 78 & $\mathrm{~K} 25$ & Gastric ulcer & 504 & $0.26 \%$ & $75.62 \%$ & $4,742 €$ \\
\hline 79 & L03 & $\begin{array}{l}\text { Cellulitis and acute } \\
\text { lymphangitis }\end{array}$ & 487 & $0.25 \%$ & $75.88 \%$ & $2,911 €$ \\
\hline 80 & A08 & $\begin{array}{l}\text { Viral and other specified } \\
\text { intestinal infections }\end{array}$ & 479 & $0.25 \%$ & $76.13 \%$ & $2,687 €$ \\
\hline 81 & $\mathrm{I} 74$ & $\begin{array}{l}\text { Arterial embolism and } \\
\text { thrombosis }\end{array}$ & 476 & $0.25 \%$ & $76.38 \%$ & $7,158 €$ \\
\hline 82 & $\mathrm{R} 10$ & Abdominal and pelvic pain & 465 & $0.24 \%$ & $76.62 \%$ & $1,478 €$ \\
\hline 83 & I95 & Hypotension & 457 & $0.24 \%$ & $76.86 \%$ & $1,802 €$ \\
\hline 84 & T81 & $\begin{array}{l}\text { Complications of procedures, } \\
\text { not elsewhere classified }\end{array}$ & 437 & $0.23 \%$ & $77.09 \%$ & $5,231 €$ \\
\hline 85 & G35 & Multiple sclerosis & 428 & $0.22 \%$ & $77.31 \%$ & $5,181 €$ \\
\hline 86 & $\mathrm{I} 20$ & Angina pectoris & 427 & $0.22 \%$ & $77.53 \%$ & $2,805 €$ \\
\hline 87 & M80 & $\begin{array}{l}\text { Osteoporosis with current } \\
\text { pathological fracture }\end{array}$ & 424 & $0.22 \%$ & $77.76 \%$ & $4,484 €$ \\
\hline 88 & J10 & $\begin{array}{l}\text { Influenza due to other } \\
\text { identified influenza virus }\end{array}$ & 423 & $0.22 \%$ & $77.98 \%$ & $3,670 €$ \\
\hline 89 & $\mathrm{R} 26$ & $\begin{array}{l}\text { Abnormalities of gait and } \\
\text { mobility }\end{array}$ & 413 & $0.22 \%$ & $78.19 \%$ & $4,670 €$ \\
\hline 90 & S02 & $\begin{array}{l}\text { Fracture of skull and facial } \\
\text { bones }\end{array}$ & 406 & $0.21 \%$ & $78.41 \%$ & $2,631 €$ \\
\hline
\end{tabular}


Table 3. Continued

\begin{tabular}{|c|c|c|c|c|c|c|}
\hline No. & $\begin{array}{l}\text { ICD-10- GM } \\
\text { (three digits) }\end{array}$ & ICD-name & Cases & $\begin{array}{l}\% \\
\text { hospital } \\
\text { cases }\end{array}$ & $\begin{array}{l}\% \\
\text { hospital } \\
\text { cases } \\
\text { (cumulated) }\end{array}$ & $\begin{array}{l}\text { Mean } \\
\text { cost per } \\
\text { hospital } \\
\text { case }\end{array}$ \\
\hline 91 & F01 & Vascular dementia & 401 & $0.21 \%$ & $78.61 \%$ & $5,352 €$ \\
\hline 92 & $\mathrm{R} 11$ & Nausea and vomiting & 395 & $0.21 \%$ & $78.82 \%$ & $1,786 €$ \\
\hline 93 & C18 & Malignant neoplasm of colon & 383 & $0.20 \%$ & $79.02 \%$ & $8,317 €$ \\
\hline 94 & K62 & $\begin{array}{l}\text { Other diseases of anus and } \\
\text { rectum }\end{array}$ & 371 & $0.19 \%$ & $79.22 \%$ & $3,256 €$ \\
\hline 95 & K26 & Duodenal ulcer & 366 & $0.19 \%$ & $79.41 \%$ & $4,268 €$ \\
\hline 96 & $\mathrm{~J} 40$ & $\begin{array}{l}\text { Bronchitis, not specified as } \\
\text { acute or chronic }\end{array}$ & 365 & $0.19 \%$ & $79.60 \%$ & $2,493 €$ \\
\hline 97 & $\mathrm{Z} 45$ & $\begin{array}{l}\text { Encounter for adjustment and } \\
\text { management of implanted } \\
\text { device }\end{array}$ & 363 & $0.19 \%$ & $79.79 \%$ & $3,835 €$ \\
\hline 98 & K83 & Other diseases of biliary tract & 338 & $0.18 \%$ & $79.96 \%$ & $3,987 €$ \\
\hline 99 & C67 & $\begin{array}{l}\text { Malignant neoplasm of } \\
\text { bladder }\end{array}$ & 334 & $0.17 \%$ & $80.14 \%$ & $3,986 €$ \\
\hline 100 & F07 & $\begin{array}{l}\text { Personality and behavioral } \\
\text { disorders }\end{array}$ & 317 & $0.17 \%$ & $80.31 \%$ & $6,327 €$ \\
\hline 101 & A49 & $\begin{array}{l}\text { Bacterial infection of } \\
\text { unspecified site }\end{array}$ & 293 & $0.15 \%$ & $80.46 \%$ & $2,985 €$ \\
\hline 102 & S01 & Open wound of head & 289 & $0.15 \%$ & $80.61 \%$ & $1,007 €$ \\
\hline 103 & R33 & Retention of urine & 275 & $0.14 \%$ & $80.75 \%$ & $1,299 €$ \\
\hline 104 & K40 & Inguinal hernia & 267 & $0.14 \%$ & $80.89 \%$ & $3,366 €$ \\
\hline 105 & $\mathrm{~S} 80$ & $\begin{array}{l}\text { Superficial injury of knee and } \\
\text { lower leg }\end{array}$ & 258 & $0.13 \%$ & $81.03 \%$ & $2,914 €$ \\
\hline 106 & Z49 & $\begin{array}{l}\text { Encounter for care involving } \\
\text { renal dialysis }\end{array}$ & 252 & $0.13 \%$ & $81.16 \%$ & $8,529 €$ \\
\hline 107 & L02 & $\begin{array}{l}\text { Cutaneous abscess, furuncle } \\
\text { and carbuncle }\end{array}$ & 215 & $0.11 \%$ & $81.27 \%$ & $3,176 €$ \\
\hline 108 & A40 & Streptococcal sepsis & 212 & $0.11 \%$ & $81.38 \%$ & $4,641 €$ \\
\hline 109 & R06 & Abnormalities of breathing & 206 & $0.11 \%$ & $81.49 \%$ & $1,265 €$ \\
\hline 110 & I44 & $\begin{array}{l}\text { Atrioventricular and left } \\
\text { bundle-branch block }\end{array}$ & 202 & $0.11 \%$ & $81.60 \%$ & $5,528 €$ \\
\hline 111 & $\mathrm{~K} 08$ & $\begin{array}{l}\text { Other disorders of teeth and } \\
\text { supporting structures }\end{array}$ & 196 & $0.10 \%$ & $81.70 \%$ & $2,362 €$ \\
\hline 112 & T17 & $\begin{array}{l}\text { Foreign body in respiratory } \\
\text { tract }\end{array}$ & 195 & $0.10 \%$ & $81.80 \%$ & $3,312 €$ \\
\hline 113 & N20 & Calculus of kidney and ureter & 192 & $0.10 \%$ & $81.90 \%$ & $3,554 €$ \\
\hline 114 & I49 & Other cardiac arrhythmias & 191 & $0.10 \%$ & $82.00 \%$ & $5,138 €$ \\
\hline 115 & $\mathrm{~S} 20$ & Superficial injury of thorax & 188 & $0.10 \%$ & $82.10 \%$ & $1,563 €$ \\
\hline 116 & I35 & $\begin{array}{l}\text { Nonrheumatic aortic valve } \\
\text { disorders }\end{array}$ & 187 & $0.10 \%$ & $82.20 \%$ & $7,909 €$ \\
\hline 117 & F32 & $\begin{array}{l}\text { Major depressive disorder, } \\
\text { single episode }\end{array}$ & 181 & $0.10 \%$ & $82.29 \%$ & $5,245 €$ \\
\hline Rest & & $\begin{array}{l}\text { Over } 455 \text { other diagnoses with } \\
\text { frequency }<0,1 \%\end{array}$ & 33,852 & $17,71 \%$ & $100 \%$ & $4,226 €$ \\
\hline \multicolumn{2}{|c|}{ Total hospitalizations } & $\begin{array}{l}\text { All hospitalization diagnoses in } \\
\text { our study population of } \\
242,236 \text { nursing home } \\
\text { residents }\end{array}$ & 191,174 & $100 \%$ & $100 \%$ & $4,030 €$ \\
\hline
\end{tabular}

*Table 3 is based on the data request (see Extended data in the data availability section). 
Table 4. Expert panel composition for the Delphi study.

\begin{tabular}{|l|l|l|l|l|}
\hline Experience & Expertise/specialization & N planned & N round 1 & N round 2 \\
\hline $\begin{array}{l}\text { Clinical, } \\
\text { physician }\end{array}$ & Primary care physicians & 30 & 31 & 29 \\
\hline $\begin{array}{l}\text { Clinical, } \\
\text { physician }\end{array}$ & Hospital specialists & 30 & 34 & 30 \\
\hline Clinical, nursing & Nursing home professionals/staff & 30 & 31 & 26 \\
\hline Research & $\begin{array}{l}\text { Medical research, pharmacology/pharmacy, } \\
\text { nursing science, health services research }\end{array}$ & 10 & 11 & 11 \\
\hline & Total & 100 & 107 & 96 \\
\hline
\end{tabular}

For 34 of the 117 ICD-10 codes, the four groups of experts gave statistically significant different preventability estimations. On average, estimations differed by only $5 \%$, and by a maximum of $15 \%$ for individual ICD-10 codes. In most of these 34 cases, the clinicians indicated slightly lower estimates. A statistically significant difference of 5\% in the median between men (median estimate at 25\%) and women (median estimate at 30\%) was found for only one of the 117 ICD-10 codes. All age groups of experts (under 40 years old $(n=29), 40-49$ years old $(n=20), 50-59$ years old $(n=25)$, and 60 years or older $(n=20))$ were consistent in their estimates of potential avoidability. For 114 of 117 ICD-10 codes the difference in the estimated proportion of potentially avoidable hospitalizations between individual age groups (median) amounted to maximum 5\%. The maximum difference found was 10\% (only for one ICD-10 code). Age groups differed in their estimates for six conditions, although for these the average assessments were only $2.5 \%$ and never more than $7.5 \%$ apart.

For 38 ICD-10 codes, the potentially avoidable hospitalization rate was estimated to be at least $75 \%$; for 12 ICD-10 codes it was estimated to be at least $90 \%$. For 35 of these 38 ICD-10 codes, the unambiguous assessment of a condition as potentially nursing home-sensitive was already clear after the second round of questioning: the range of dispersion around the median of three quarters of all expert's assessments for the respective ICD-10 code was 15\% or less. The three for which the latter did not apply were discussed at the expert workshop. Further, a total of 22 ICD-10 codes had a median preventability proportion below $75 \%$, but their scatter spectrum for three quarters of all assessments contained the relevant preventability proportion of $75 \%$, signaling a relevant but still ambiguous preventability proportion. Thus, a total of 25 ICD-10 codes were prepared for the expert workshop consensus process (Table 5; ICD-10 codes discussed in workshop are shown in italic font).

Overall, after the workshop with 16 experts, all proportions for potentially avoidable hospitalizations could be corroborated, and 58 ICD-10 codes with an estimated potential avoidability of at least $70 \%$, were selected for the list of nursing home-sensitive conditions. In Table 5 the nursing home-sensitive conditions are shown in the greyed table part. Table 6 shows these conditions sorted by disease category.

\section{Comparison of nursing home-sensitive with ambulatory care-sensitive conditions}

For the comparison of nursing home-sensitive diagnoses with ambulatory care-sensitive diagnoses, the occurrence of the 117 ICD-10 codes in the ambulatory care-sensitive groups was reviewed. In Table 5, the last three columns were added to show the results of this review. Comparing the 58 nursing home-sensitive conditions with the ambulatory care-sensitive conditions, it appeared that only 28 three-digit and seven four-digit nursing home-sensitive ICD-10 conditions were also included in the ambulatory care-sensitive ones. Therefore, $60 \%(35 / 58)$ of the nursing home-sensitive conditions were also partly or completely ambulatory care-sensitive, and $40 \%$ were not.

Extending this comparison to all common conditions in nursing home residents, only 29 three-digit and another 10 fourdigit of the 117 ICD-10 codes were ambulatory care-sensitive. Thus, only 33\% (39/117) of the ICD-10 codes relevant to the nursing home population were partially or wholly ambulatory care-sensitive, and $67 \%$ were not.

Sundmacher published preventability estimates for the core ambulatory care-sensitive groups of ICD-10 codes. A total of $27(47 \%)$ nursing home-sensitive conditions belong to these core ambulatory care-sensitive conditions ( 23 completely [three-digit ICD-10] and four only partially [four-digit ICD-10]), and 53\% do not. Of all 117 ICD-10 codes, 31 (26\%) conditions appeared partly (seven four-digit ICD-10 codes) or wholly (24 three-digit ICD-10 codes) in this core list, and $74 \%$ do not. 


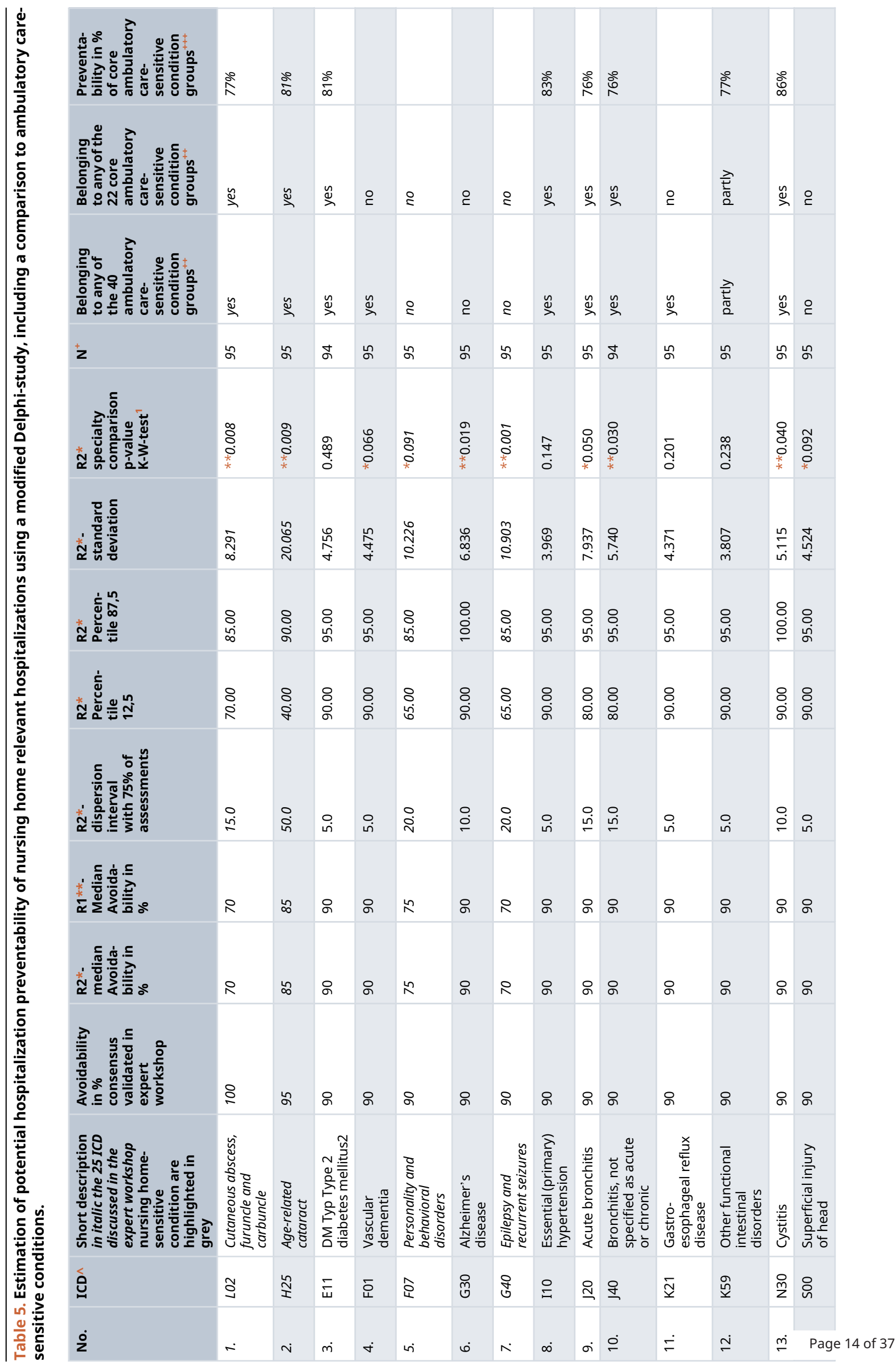




\begin{tabular}{|c|c|c|c|c|c|c|c|c|c|c|c|c|c|c|}
\hline 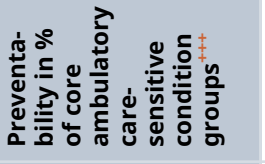 & & & ஓे & ڤे & ஓे & ๙े & & 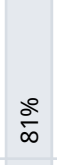 & & ڤे & & & 今̊ & ڤั \\
\hline 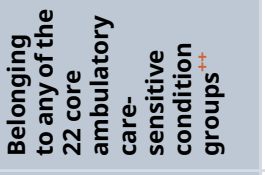 & $\stackrel{\circ}{\circ}$ & $\stackrel{\circ}{\circ}$ & $\stackrel{\check{\beth}}{\supseteq}$ & $\stackrel{\check{x}}{\check{\nu}}$ & $\stackrel{\swarrow}{\curvearrowright}$ & $\stackrel{\searrow}{\beth}$ & ๕ & $\stackrel{\check{\Perp}}{\curvearrowright}$ & 尺 & 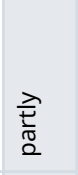 & $\stackrel{\circ}{\circ}$ & $\stackrel{\circ}{\circ}$ & $\beth_{\beth}^{\searrow}$ & $\stackrel{\check{x}}{\check{\nu}}$ \\
\hline 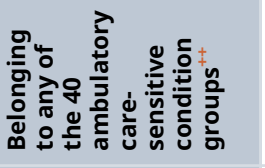 & $\stackrel{\circ}{\check{1}}$ & $\stackrel{\circ}{\check{1}}$ & $\stackrel{\tilde{x}}{\check{\nu}}$ & 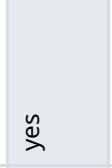 & $\stackrel{\check{\nu}}{\curvearrowright}$ & 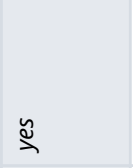 & 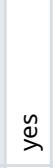 & $\stackrel{\check{\nu}}{\check{\nu}}$ & 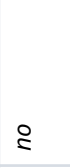 & 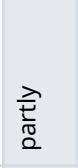 & $\stackrel{\circ}{\circ}$ & $\stackrel{\circ}{\circ}$ & $\stackrel{\coprod}{\beth}$ & $\stackrel{\check{\nu}}{\curvearrowright}$ \\
\hline$z$ & ณ & ณn & ณn & ณ & ณ & n & ณ & น & n & ณn & के & นૂ & n & ณ \\
\hline 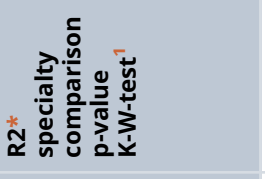 & 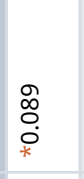 & $\stackrel{\hat{n}}{\frac{n}{0}}$ & $\begin{array}{l}m \\
\substack{\infty \\
0 \\
0}\end{array}$ & $\stackrel{\bar{\sigma}}{\circ}$ & ồ & \begin{tabular}{l}
$\overline{0}$ \\
0 \\
0 \\
\multirow{x}{*}{}
\end{tabular} & 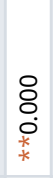 & 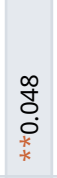 & 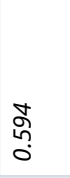 & 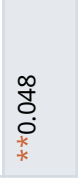 & $\stackrel{\infty}{\underset{0}{0}}$ & $\frac{m}{0}$ & $\begin{array}{l}\Delta \\
\vdots \\
0\end{array}$ & 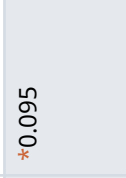 \\
\hline 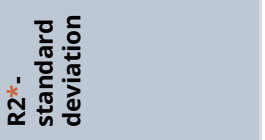 & $\begin{array}{l}\text { R } \\
\text { ô } \\
\text { in }\end{array}$ & $\begin{array}{l}\infty \\
\infty \\
\infty \\
\text { in }\end{array}$ & $\begin{array}{l}\text { ô } \\
\text { ò } \\
\text { in }\end{array}$ & $\stackrel{\stackrel{\sim}{\sim}}{\underset{ٍ}{0}}$ & 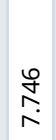 & 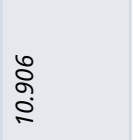 & $\begin{array}{l}\bar{o} \\
\dot{0}\end{array}$ & $\begin{array}{l}\hat{\hat{O}} \\
\stackrel{+}{+}\end{array}$ & $\begin{array}{l}\stackrel{R}{f} \\
\infty\end{array}$ & 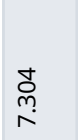 & $\begin{array}{l}\hat{y} \\
\text { ò }\end{array}$ & 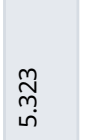 & $\begin{array}{l}\underset{J}{J} \\
\text { }\end{array}$ & $\stackrel{m}{\stackrel{m}{N}}$ \\
\hline 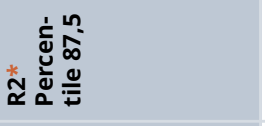 & $\begin{array}{l}\text { ㅇ․ } \\
\text { ஸ் }\end{array}$ & $\begin{array}{l}8 \\
\text { ஸें }\end{array}$ & 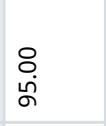 & $\begin{array}{l}\text { 오. } \\
\text { ڤે }\end{array}$ & $\begin{array}{l}\text { 어 } \\
\text { گू }\end{array}$ & $\begin{array}{l}8 \\
\text { \& }\end{array}$ & $\begin{array}{l}\text { 오 } \\
\text { ஸे }\end{array}$ & $\begin{array}{l}\text { ㅇ․ } \\
\text { ஸे }\end{array}$ & $\begin{array}{l}\stackrel{0}{0} \\
\infty\end{array}$ & $\begin{array}{l}8 \\
\text { گૂં }\end{array}$ & $\begin{array}{l}\text { m̊. } \\
\text { ه̊ }\end{array}$ & $\begin{array}{l}\text { ஃ̊ } \\
\text { ه̊ }\end{array}$ & $\begin{array}{l}\stackrel{0}{0} \\
\infty\end{array}$ & $\begin{array}{l}\text { ஃ } \\
\text { ه̊ }\end{array}$ \\
\hline 离 & $\begin{array}{l}8 \\
8 \\
8\end{array}$ & $\begin{array}{l}8 \\
8 \\
8\end{array}$ & $\begin{array}{l}\stackrel{8}{0} \\
\text { \&. }\end{array}$ & $\begin{array}{l}\text { \& } \\
\text { ळ. }\end{array}$ & $\begin{array}{l}\text { வ } \\
\text { क }\end{array}$ & $\begin{array}{l}8 \\
\stackrel{8}{\aleph}\end{array}$ & $\begin{array}{l}\text { ¿े } \\
\infty\end{array}$ & $\begin{array}{l}\text { ¿े. } \\
\text { ळ. }\end{array}$ & $\begin{array}{l}8 \\
\text { î }\end{array}$ & $\begin{array}{l}8 \\
\text { \&. } \\
\infty\end{array}$ & $\begin{array}{l}\stackrel{\infty}{m} \\
\stackrel{\Gamma}{R}\end{array}$ & $\begin{array}{l}\text { \& } \\
\text { ஸे }\end{array}$ & $\begin{array}{l}8 \\
\text { iి } \\
6\end{array}$ & $\begin{array}{l}\text { \& } \\
\text { ri }\end{array}$ \\
\hline 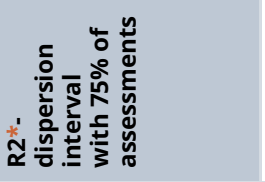 & 우 & 우 & $\stackrel{\circ}{\circ}$ & $\stackrel{\circ}{\circ}$ & 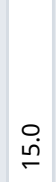 & $\stackrel{\circ}{\stackrel{\text { i }}{ }}$ & مُ & $\stackrel{\circ}{\circ}$ & ئ & $\stackrel{\circ}{\circ}$ & $\stackrel{m}{\stackrel{m}{F}}$ & 우 & 占 & $\stackrel{\text { ம் }}{\stackrel{2}{ }}$ \\
\hline 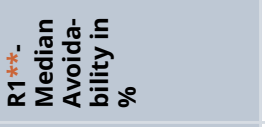 & 8 & ৪ & $\stackrel{\llcorner}{\infty}$ & 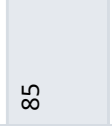 & $\mathscr{\infty}$ & 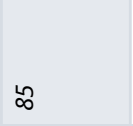 & $\stackrel{\leftrightarrow}{\infty}$ & $\stackrel{\curvearrowleft}{\infty}$ & $R$ & $\stackrel{\Perp}{\infty}$ & 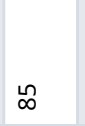 & œ & 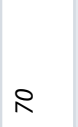 & $\infty$ \\
\hline 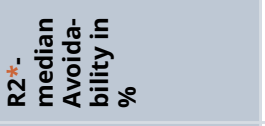 & 8 & 8 & $\stackrel{\infty}{\infty}$ & 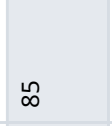 & $\stackrel{\llcorner}{\infty}$ & 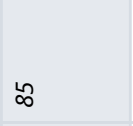 & $\stackrel{\infty}{\infty}$ & $\stackrel{\llcorner}{\infty}$ & 尺 & 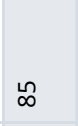 & 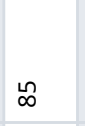 & $\stackrel{\Perp}{\infty}$ & $R$ & 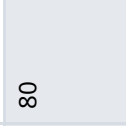 \\
\hline 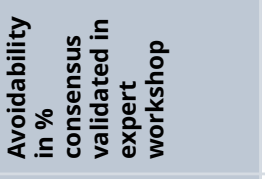 & ৪ & ৪ & $\stackrel{\llcorner}{\infty}$ & $\stackrel{\llcorner}{\infty}$ & $\stackrel{\llcorner}{\infty}$ & 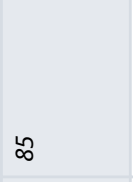 & 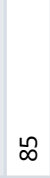 & $\stackrel{\llcorner}{\infty}$ & $\infty$ & $\stackrel{\llcorner}{\infty}$ & $\stackrel{\llcorner}{\infty}$ & $\stackrel{\llcorner}{\infty}$ & ळ & $\infty$ \\
\hline 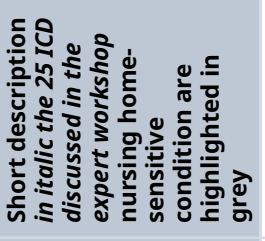 & 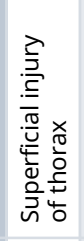 & 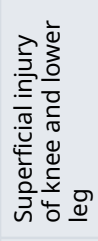 & 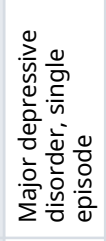 & 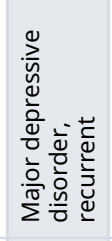 & 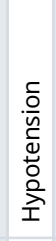 & 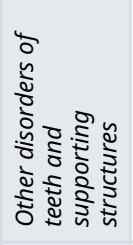 & 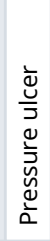 & $\begin{array}{l}\frac{\pi}{0} \\
\frac{0}{0} \\
\frac{0}{0} \\
\frac{0}{0} \\
0\end{array}$ & 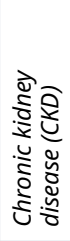 & 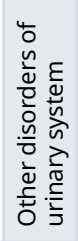 & 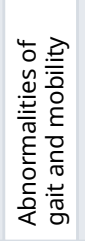 & 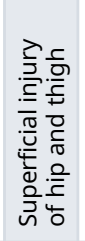 & 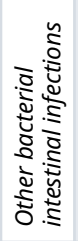 & 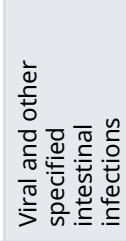 \\
\hline ئ & ్ㅗ & $\stackrel{\infty}{\infty}$ & $\tilde{\tilde{L}}$ & $\tilde{\tilde{L}}$ & $\stackrel{\text { ڤ }}{\varrho}$ & $\stackrel{\infty}{2}$ & $\stackrel{\mathscr{\Xi}}{ }$ & 莸 & $\sum^{\infty}$ & $\stackrel{\dddot{m}}{z}$ & 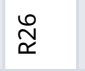 & $\stackrel{\circ}{\hat{n}}$ & ষ্ণ & $\stackrel{\infty}{\propto}$ \\
\hline$\dot{z}$ & $\ddot{n}$ & $\stackrel{\dot{\sigma}}{ }$ & $\stackrel{\circ}{\check{2}}$ & $\stackrel{\infty}{\sim}$ & $\stackrel{\circ}{\sigma}$ & ஓं & $\dot{\bar{N}}$ & $\dot{N}$ & $\stackrel{\sim}{\sim}$ & $\stackrel{\sim}{\sim}$ & ம் & $\dot{\sim}$ & $\stackrel{\sim}{i}$ & $\stackrel{\infty}{\sim}$ \\
\hline
\end{tabular}




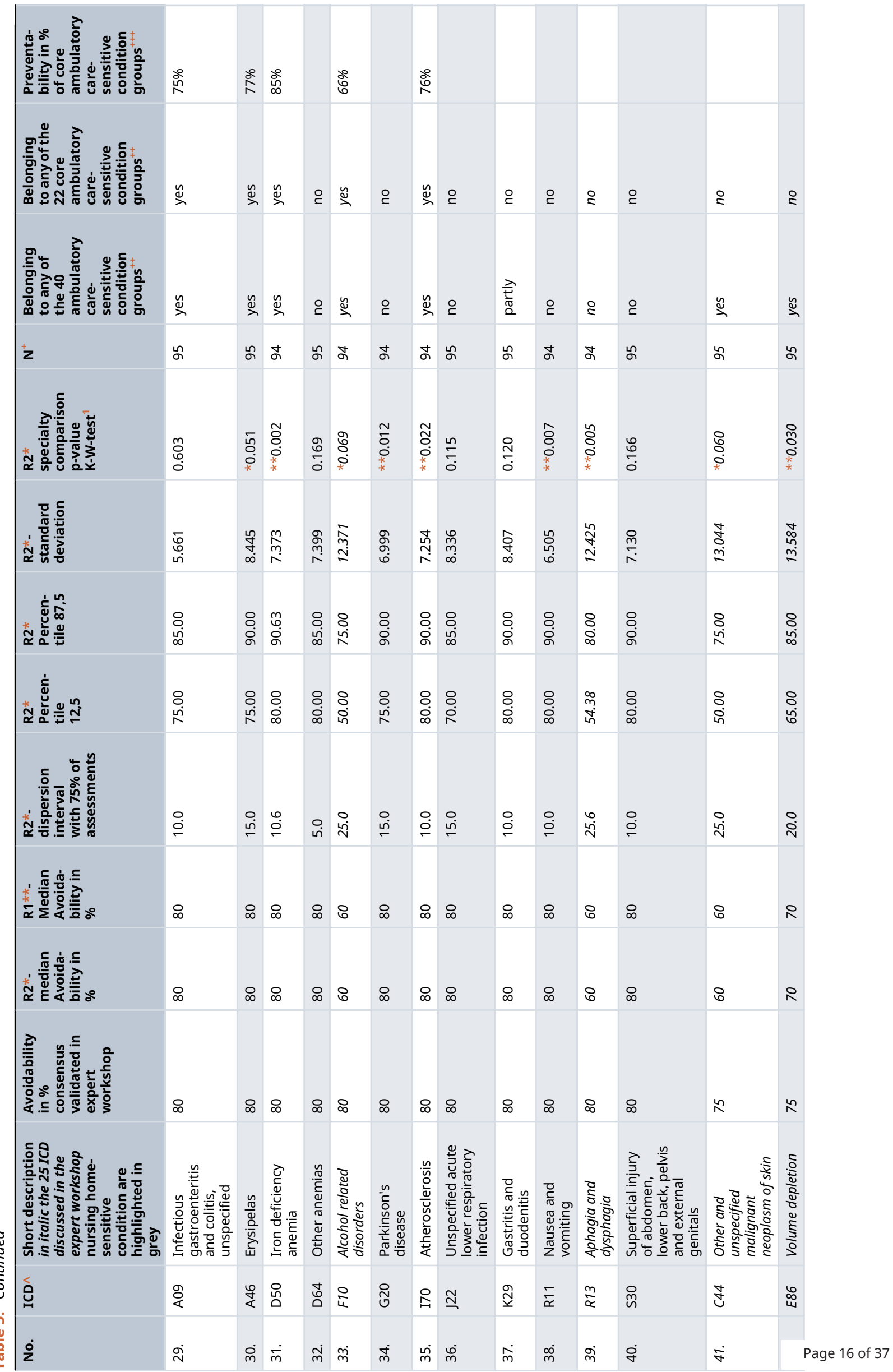




\begin{tabular}{|c|c|c|c|c|c|c|c|c|c|c|c|c|c|}
\hline 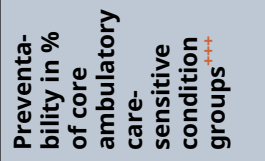 & & & & & वें & ஓ̊ & ळे & ஓ̊ & & & ફ̊ & $\stackrel{\circ}{\grave{1}}$ & \\
\hline 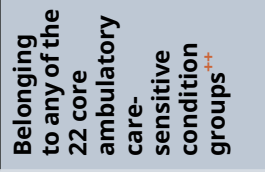 & 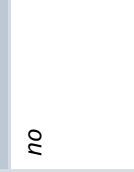 & ̊ & 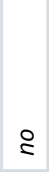 & $\stackrel{\circ}{9}$ & ๗ & डे & $\stackrel{\beth}{\beth}$ & $\stackrel{\check{\nu}}{\check{\nu}}$ & 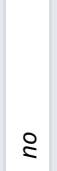 & 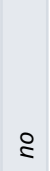 & 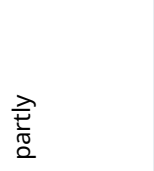 & $\stackrel{\check{\nu}}{\stackrel{\Perp}{n}}$ & 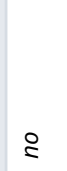 \\
\hline 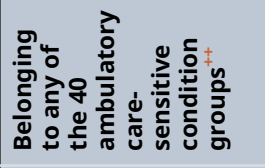 & 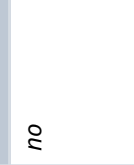 & 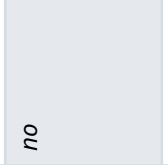 & 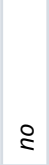 & $\stackrel{\circ}{ }$ & 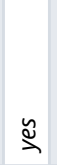 & $\begin{array}{l}\overrightarrow{\mathrm{t}} \\
\text { हे }\end{array}$ & $\stackrel{\check{\beth}}{\check{n}}$ & $\stackrel{\breve{\nu}}{\check{\lambda}}$ & $\begin{array}{l}\text { ते } \\
\text { जे }\end{array}$ & $\cong$ & $\begin{array}{l}\text { 屁 } \\
\frac{1}{0}\end{array}$ & $\stackrel{\check{\nu}}{\check{\nu}}$ & 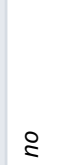 \\
\hline$z$ & nू & n & no & นั & ณ & nू & nू & นૂ & nू & ผn & nू & ณ & n \\
\hline 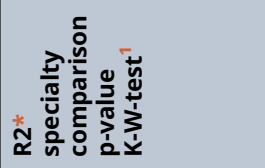 & $\underset{0}{\stackrel{n}{0}}$ & $\begin{array}{c}\stackrel{0}{0} \\
\stackrel{m}{0}\end{array}$ & 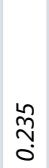 & \begin{tabular}{l} 
के \\
0 \\
0 \\
\multirow{2}{*}{}
\end{tabular} & $\begin{array}{l}\hat{\stackrel{o}{ }} \\
\dot{0} \\
\dot{x}\end{array}$ & $\begin{array}{l}\bar{\delta} \\
\stackrel{0}{x} \\
\dot{x}\end{array}$ & 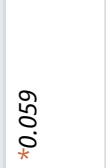 & $\begin{array}{l}0 \\
\vdots \\
0 \\
0 \\
* \\
*\end{array}$ & $\underset{\mathrm{n}}{\mathrm{i}}$ & 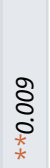 & \begin{tabular}{l}
$\overline{0}$ \\
$\dot{0}$ \\
\multirow{*}{*}{}
\end{tabular} & $\begin{array}{l}\hat{\tilde{o}} \\
\dot{0} \\
\dot{x}\end{array}$ & $\begin{array}{l}\bar{N} \\
\text { }\end{array}$ \\
\hline 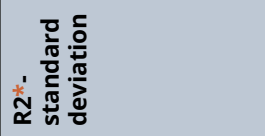 & $\begin{array}{l}0 \\
0 \\
0 \\
0 \\
0\end{array}$ & $\begin{array}{l}\text { ळ } \\
\stackrel{2}{\circ} \\
\stackrel{2}{0}\end{array}$ & 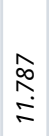 & $\begin{array}{l}\stackrel{\vartheta}{0} \\
\infty\end{array}$ & $\begin{array}{l}\text { ठे } \\
\text { هे }\end{array}$ & $\begin{array}{l}\frac{0}{n} \\
\stackrel{n}{n}\end{array}$ & 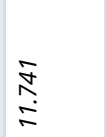 & $\begin{array}{l}\infty \\
0 \\
0 \\
\infty\end{array}$ & $\begin{array}{l}\stackrel{J}{N} \\
\stackrel{n}{n}\end{array}$ & 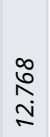 & 号 & 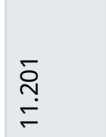 & $\underset{\substack{\hat{\infty} \\
\stackrel{N}{N}}}{ }$ \\
\hline 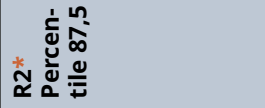 & 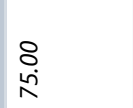 & $\begin{array}{l}\stackrel{8}{0} \\
\infty\end{array}$ & 离 & $\begin{array}{l}\text { \&. } \\
\text { w. }\end{array}$ & $\begin{array}{l}8 \\
\stackrel{1}{N}\end{array}$ & $\begin{array}{l}8 \\
\text { \& }\end{array}$ & $\begin{array}{l}\stackrel{8}{0} \\
\infty\end{array}$ & 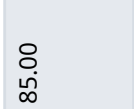 & $\begin{array}{l}8 \\
\text { \& } \\
\end{array}$ & $\begin{array}{l}8 \\
\text { \& }\end{array}$ & $\begin{array}{l}\text { ¿ } \\
\text { in }\end{array}$ & $\begin{array}{l}\text { : } \\
\text { هं }\end{array}$ & $\begin{array}{l}8 \\
\infty \\
\infty\end{array}$ \\
\hline 离 & $\begin{array}{l}8 \\
\text { wi } \\
\text { m. }\end{array}$ & $\begin{array}{l}8 \\
\stackrel{0}{0}\end{array}$ & $\begin{array}{l}8 \\
\text { ì }\end{array}$ & : & $\begin{array}{l}8 \\
\stackrel{0}{0}\end{array}$ & $\begin{array}{l}8 \\
\stackrel{8}{n}\end{array}$ & $\begin{array}{l}8 \\
\text { 出 }\end{array}$ & $\stackrel{\text { ¿ }}{\circ}$ & $\begin{array}{l}8 \\
\text { in } \\
\text { n }\end{array}$ & $\begin{array}{l}8 \\
8 \\
0\end{array}$ & $\stackrel{\circ}{\stackrel{\circ}{R}}$ & $\begin{array}{l}8 \\
\stackrel{1}{n}\end{array}$ & $\begin{array}{l}8 \\
\stackrel{0}{0}\end{array}$ \\
\hline 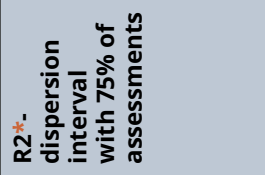 & 过 & ஓ्. & ฆे & 官 & i̊ & ڤ્. & ڤ્. & ف요 & $\stackrel{\stackrel{\sim}{\sim}}{ }$ & 占 & 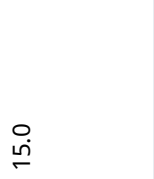 & 울 & ஓ̊. \\
\hline 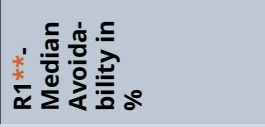 & in & $\stackrel{R}{R}$ & in & $\stackrel{n}{\kappa}$ & 8 & 织 & 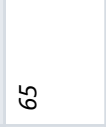 & $\stackrel{n}{\kappa}$ & 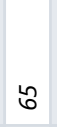 & ใ̊ & n & $\stackrel{n}{\kappa}$ & R \\
\hline 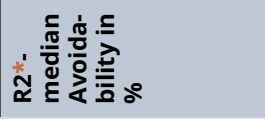 & in & $\stackrel{R}{R}$ & 台 & $\stackrel{n}{\kappa}$ & 8 & $\kappa_{n}^{n}$ & 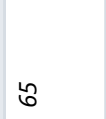 & $\stackrel{n}{\kappa}$ & 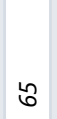 & ใ & $\stackrel{n}{n}$ & $\stackrel{n}{\kappa}$ & $R$ \\
\hline 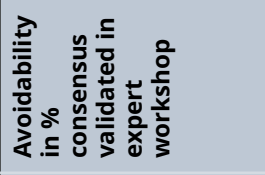 & $\stackrel{n}{\kappa}$ & $\stackrel{n}{\wedge}$ & $\stackrel{n}{\kappa}$ & $\stackrel{n}{R}$ & $\stackrel{n}{N}$ & $\stackrel{n}{\wedge}$ & $\stackrel{n}{\wedge}$ & $\stackrel{n}{N}$ & $\stackrel{n}{\kappa}$ & $\stackrel{\kappa}{\kappa}$ & $\stackrel{n}{\wedge}$ & $\stackrel{\mathfrak{n}}{\wedge}$ & $\stackrel{n}{\kappa}$ \\
\hline 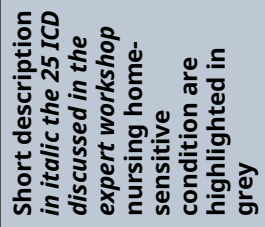 & 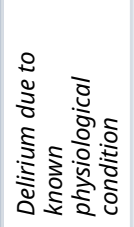 & 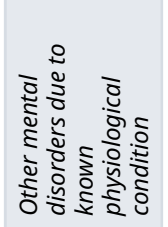 & 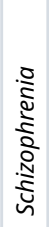 & 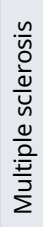 & 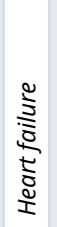 & 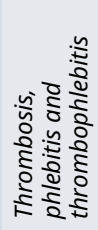 & 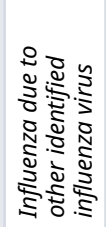 & 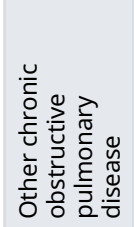 & 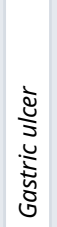 & 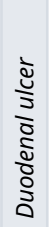 & 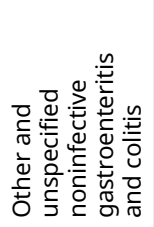 & 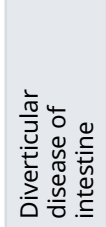 & 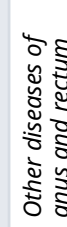 \\
\hline : & $\stackrel{2}{0}$ & \& & ¿্ষ & 岗 & 号 & 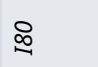 & $\stackrel{ }{=}$ & 寻 & $\stackrel{\varpi}{Ð}$ & ঙ̊ & 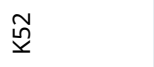 & 占 & $\stackrel{\widetilde{V}}{2}$ \\
\hline$\dot{i}$ & $\ddot{\vartheta}$ & $\mathbb{J}$ & 守 & $\dot{\sigma}$ & $\dot{\jmath}$ & $\stackrel{\infty}{\sigma}$ & gे & ம் & in & กิ่ & ભું & ஸ் & \\
\hline
\end{tabular}




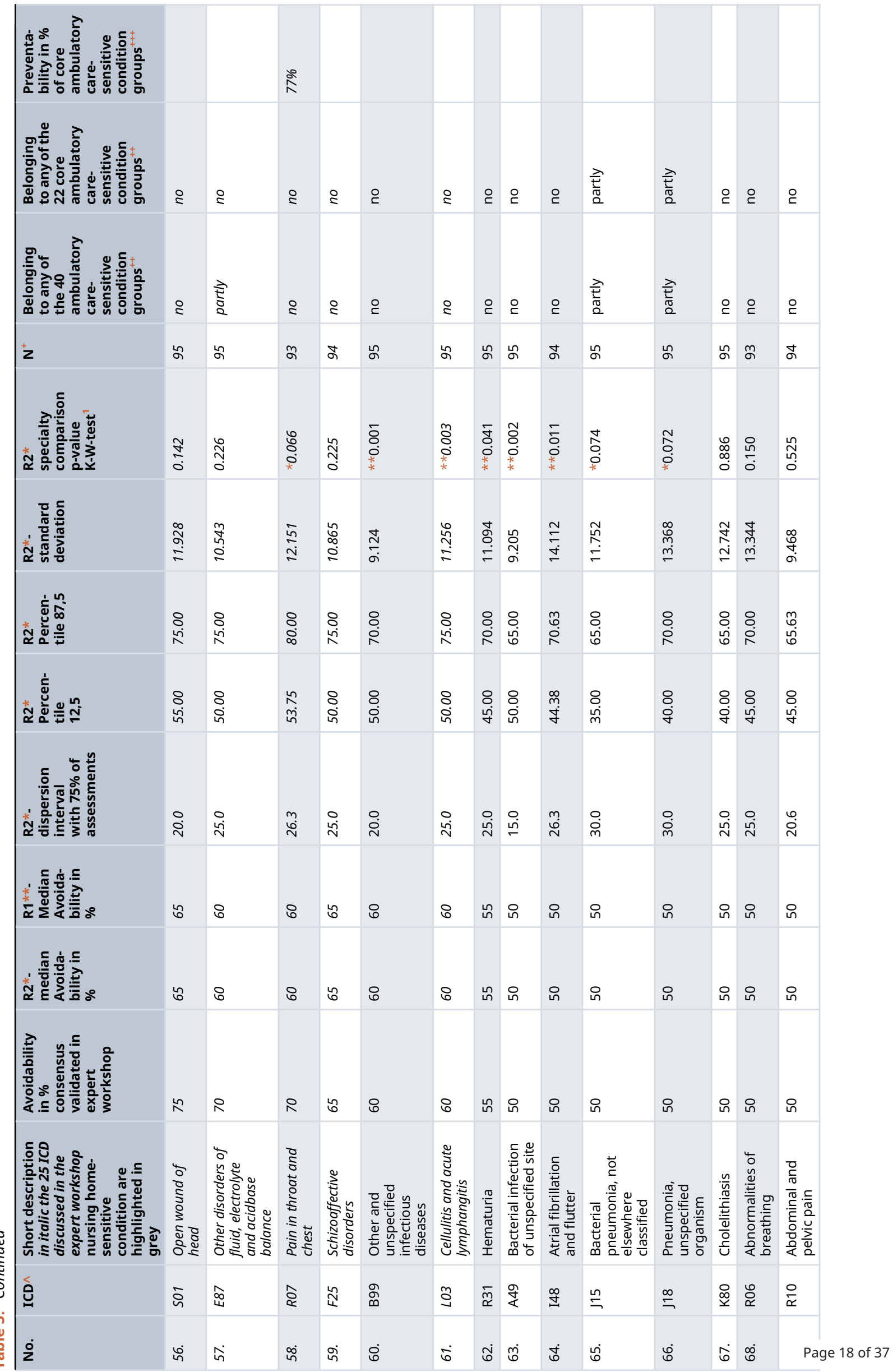




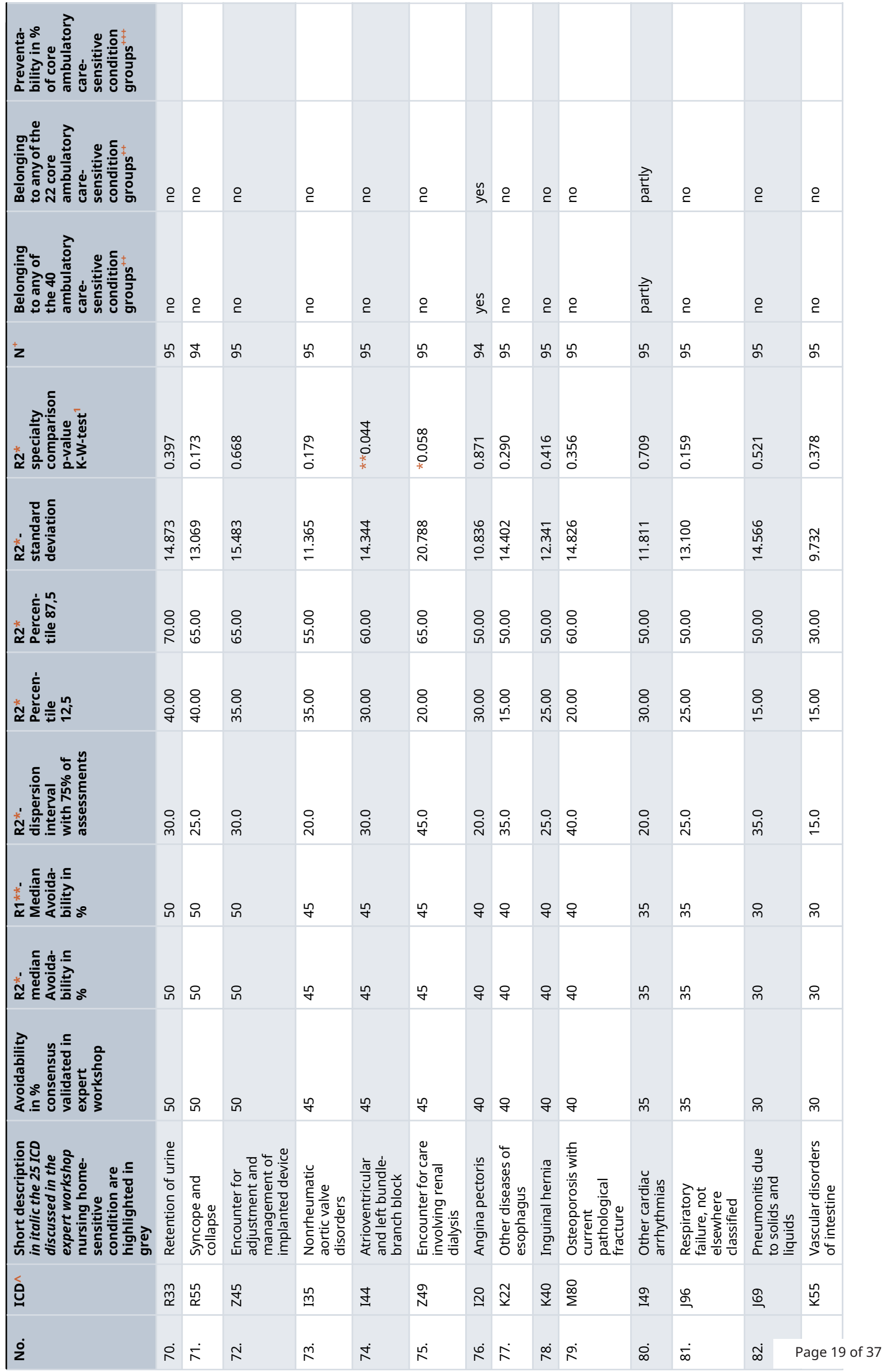




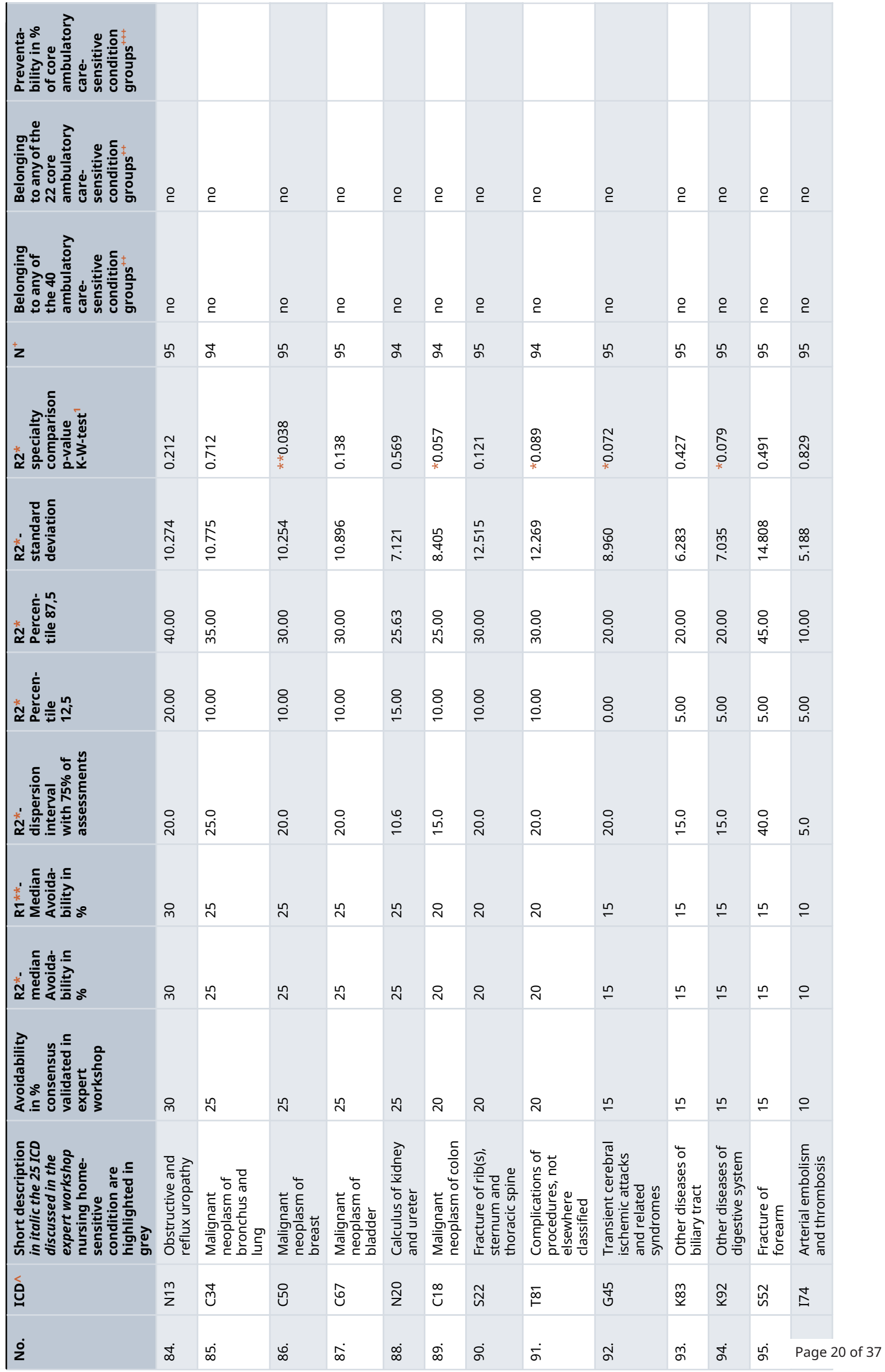




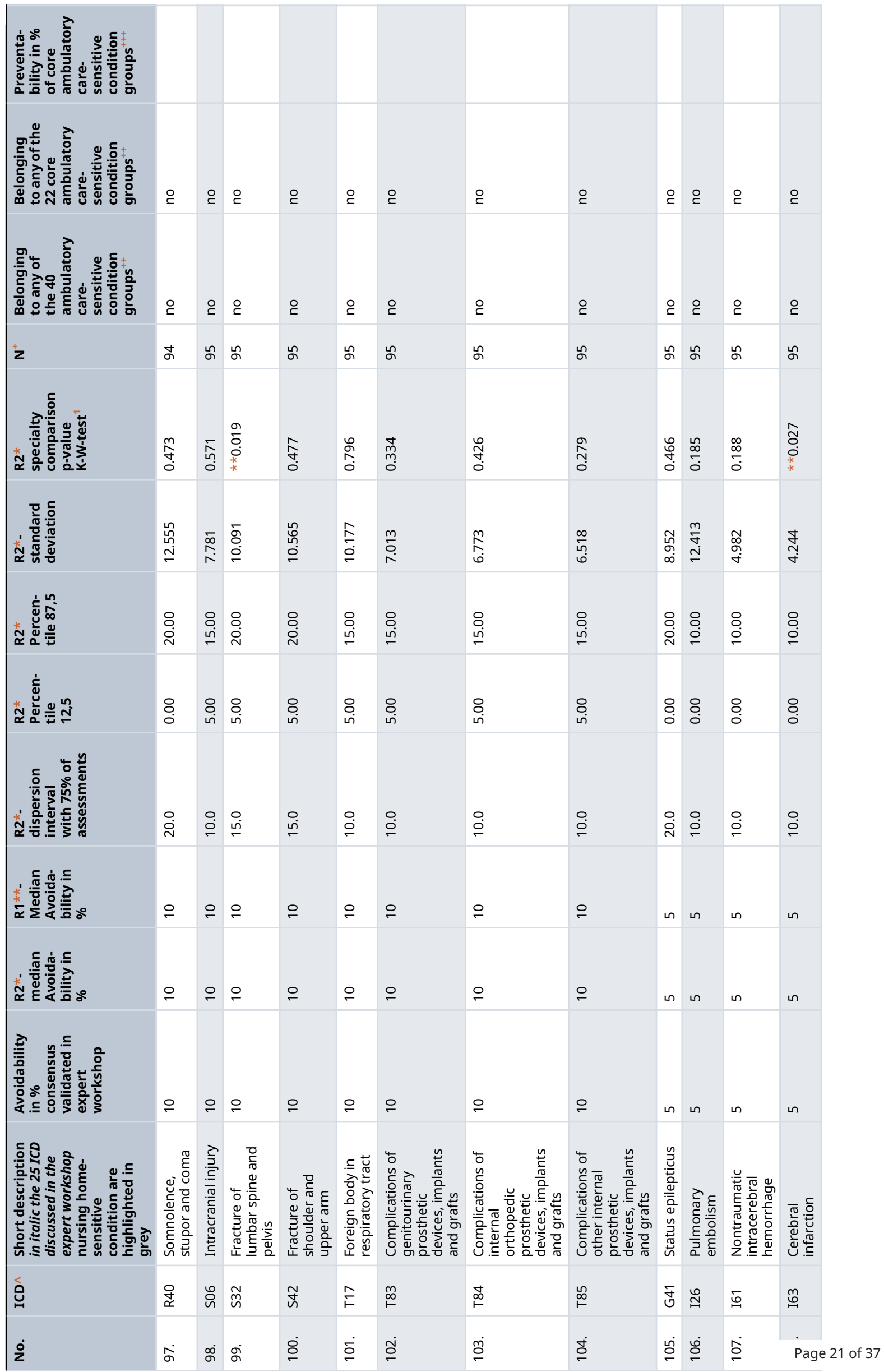




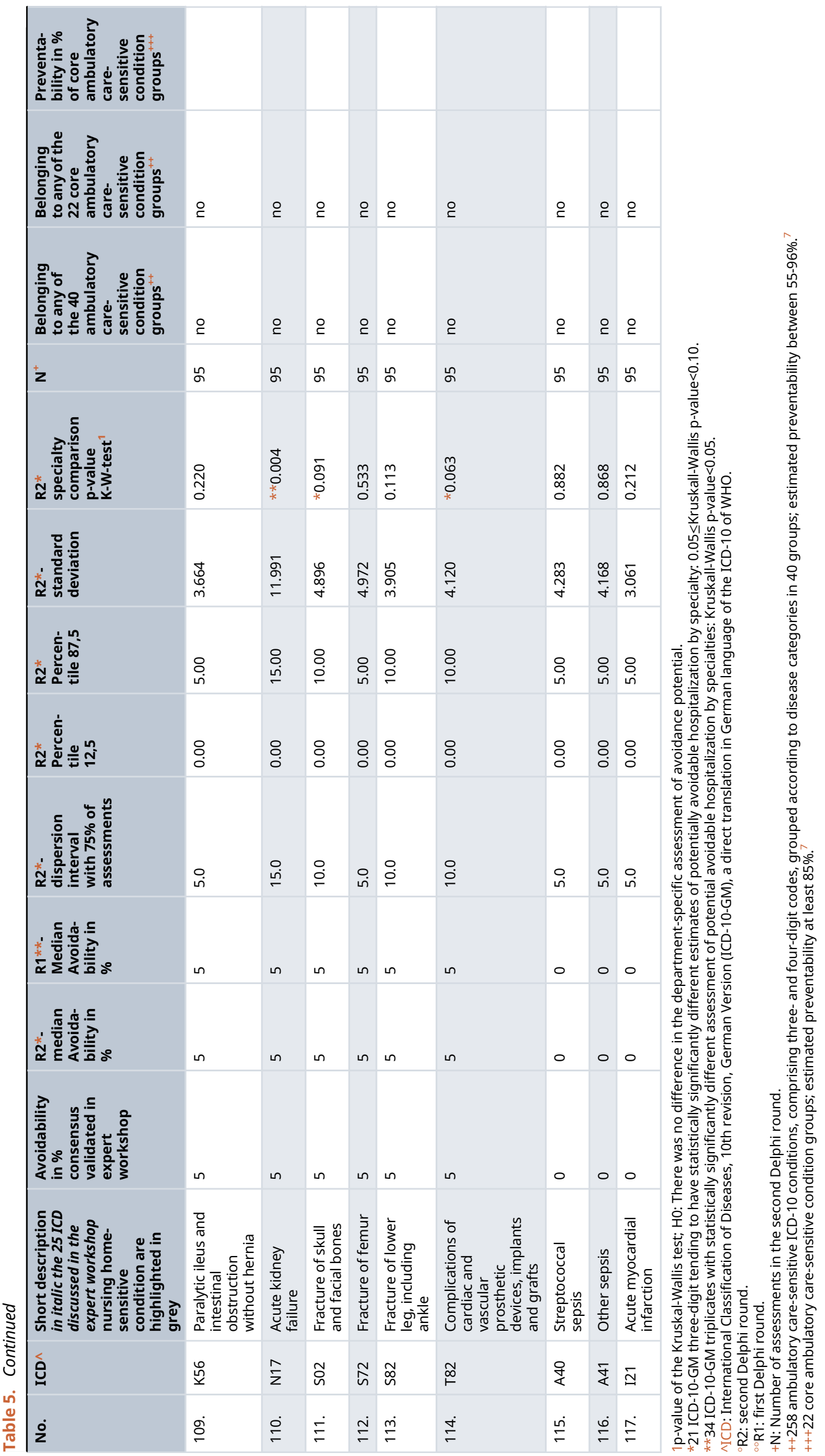


Table 6 . List of nursing home-sensitive hospital conditions sorted by disease category.

\begin{tabular}{|c|c|c|c|}
\hline No. & Condition of nursing home-sensitive hospital admission & ICD-10 codes $^{\wedge}$ & $\begin{array}{l}\text { Number } \\
\text { of ICDs^ }\end{array}$ \\
\hline 1 & Diabetes Mellitus Type 2 & E11 & 1 \\
\hline 2 & $\begin{array}{l}\text { Volume depletion and other disorders of electrolyte and acid-base } \\
\text { balance }\end{array}$ & E86, E87 & 2 \\
\hline 3 & $\begin{array}{l}\text { Gastrointestinal ulcers and inflammation, esophageal reflux } \\
\text { disease, functional bowel disorders, dysphagia }\end{array}$ & $\begin{array}{l}\text { K08, K21, K25, K26, } \\
\text { K29, K52, K57, K59, } \\
\text { K62, R13 }\end{array}$ & 10 \\
\hline 4 & Intestinal infections & $A 04, A 08, A 09, R 11$ & 4 \\
\hline 5 & Chronic kidney disease, cystitis, other diseases of the urinary system. & N18, N30, N39 & 3 \\
\hline 6 & $\begin{array}{l}\text { Dementia (vascular or Alzheimer's disease, primary Parkinson's } \\
\text { syndrome) }\end{array}$ & F01, G20, G30 & 3 \\
\hline 7 & $\begin{array}{l}\text { Mental disorders, personality or behavioral disorders, depressive } \\
\text { disorder, schizoaffective disorder and schizophrenia, delirium }\end{array}$ & $\begin{array}{l}\text { F05, F06, F07, F10, } \\
\text { F20, F32, F33 }\end{array}$ & 7 \\
\hline 8 & Other diseases of the nervous system (MS, epilepsy) & G35, G40 & 2 \\
\hline 9 & Cataracta Senilis & $\mathrm{H} 25$ & 1 \\
\hline 10 & Anemias of different origin & D50, D64 & 2 \\
\hline 11 & $\begin{array}{l}\text { Hypertension, hypotension, atherosclerosis, heart failure, } \\
\text { thrombosis, phlebitis and thrombophlebitis. }\end{array}$ & $\begin{array}{l}\text { I10, I50, I70, I80, } \\
\text { I95 }\end{array}$ & 5 \\
\hline 12 & $\begin{array}{l}\text { Other acute/chronic lower respiratory tract infections and influenza, } \\
\text { sore throat and chest pain. }\end{array}$ & $\begin{array}{l}\mathrm{J} 10, \mathrm{~J} 20, \mathrm{~J} 22, \mathrm{~J} 40, \\
\mathrm{~J} 44, \mathrm{R} 07\end{array}$ & 6 \\
\hline 13 & $\begin{array}{l}\text { Skin infections, decubital ulcer and pressure zone, skin cancer } \\
\text { exclusive melanoma }\end{array}$ & A46, C44, L02, L89 & 4 \\
\hline 14 & Back pain, disturbances of gait and mobility & M54, R26 & 2 \\
\hline 15 & Superficial injuries of various parts of the body & $\begin{array}{l}\text { S00, S01, S20, S30, } \\
\text { S70, S80 }\end{array}$ & 6 \\
\hline & \multicolumn{2}{|c|}{ Total number of nursing home sensitive conditions } & 58 \\
\hline
\end{tabular}

AICD: International Classification of Diseases, 10th revision, German Version (ICD-10-GM), a direct translation in German language of the ICD-10 of WHO.

On the other hand, 178 of the 258 ambulatory care-sensitive hospitalizations (three- and four-digit level) were not listed in the nursing home-sensitive list $(69 \%)$.

The preventability estimates were only known to the authors for the core ambulatory care-sensitive condition groups. Therefore, for only 31 out of 117 ICD-10 common nursing home hospitalizations, the preventability potential for the nursing home common as well as nursing home-sensitive ICD-10 codes were compared to their counterpart in the core ambulatory care-sensitive groups. For the 27 nursing home-sensitive conditions, the minimum and maximum differences between both settings was $-9 \%$ and $+23 \%$, respectively. For 12 ICD-10 codes, the preventability potentials of both lists were only $5 \%$ apart. For the remaining four nursing home common conditions, the difference was $-18 \%$ to $-41 \%$ apart.

Thus, our results show that nursing home-sensitive conditions are to be distinguished from ambulatory care-sensitive conditions: both diagnoses and preventability estimates differed between the settings.

\section{Extrapolation of costs of potentially avoidable hospitalizations}

In 2017, there were 3.4 million persons in need of long-term care in Germany, of which 818,289 were nursing home residents. ${ }^{28}$ With 242,236 insured persons in inpatient care, our study population represented about $29.6 \%$ of all nursing home residents in Germany. Our sample yielded 191,174 hospital cases in one year. The annual incidence was therefore around 0.79 hospital cases per insured person in stationary care $(191,174 / 242,236)$. Table 7 shows the extrapolation of the results from the analysis of routine health insurance data for Germany. For this purpose, the total costs per ICD-10 code were first calculated, weighted according to their proportion of cases (number of cases*cost per hospital case). The total cost for each ICD-10 code was than multiplied by the extrapolation factor and summed to obtain the total costs incurred in Germany. Calculating the costs for nursing home-sensitive conditions was done accordingly. Total costs per nursing home-sensitive condition were multiplied by the proportion (in \%) of potentially avoidable hospitalizations agreed to 


\section{Discussion}

Key findings

We used routine health insurance data from 242,236 nursing home residents to assess frequencies and costs of hospital admissions amongst nursing home residents; we identified 117 hospital discharge diagnoses which had a frequency rate of at least $0.1 \%$. In a two-stage Delphi study, 107 and 96 experts in round 1 and 2, respectively, estimated the potential to avoid a hospitalization for these diagnoses. After two Delphi rounds and an expert workshop, we were able to identify 58 diagnoses considered to be nursing home-sensitive conditions in the context of the German health care system. The frequency of hospital admissions for these diagnoses and associated costs to the care system were substantial, and warrant further discussion on strategies to decrease hospitalizations for these diagnoses.

\section{Comparison to other studies}

There are few comparable studies on nursing home-sensitive hospitalizations. Most of the research in this field focused on ambulatory care-sensitive hospital admissions, which cannot be directly applied to the nursing home context as the nursing home population differs regarding frequency of diseases, healing process, nursing care and systematic care conditions. However, the seminal studies by Purdy et al., ${ }^{5}$ Ouslander et al. ${ }^{8}$ and Walker et al. ${ }^{17}$ are noteworthy, for developing the concept of avoidable hospital admissions and using various methods established in health services research, from medical chart reviews to analysis of administrative datasets, to quantify the impact of avoidable hospital admissions, and indicate that a large proportion of hospital activity might in fact be avoidable. For the German health system context, Leutgeb et al. ${ }^{10}$ demonstrated that hospital admission rates are much higher amongst nursing home residents compared to community-dwelling residents.

The number of people in need of nursing home care has steadily increased over $17 \%$ in the last ten years in Germany and, considering the country's population is aging, it is expected to increase further. ${ }^{29}$ Similar trends hold true for other highincome countries. ${ }^{30}$

This is the first study to compare nursing home-sensitive with ambulatory care-sensitive conditions for the German setting. It showed that nursing home-sensitive conditions are to be distinguished from ambulatory care-sensitive conditions: $74 \%$ and 53\% of common nursing home and nursing home-sensitive conditions, respectively, do not appear on the ambulatory care-sensitive list; for the core ambulatory care-sensitive list these numbers were $67 \%$ and $40 \%$, respectively. In contrast, $69 \%$ of all ambulatory care-sensitive conditions did not appear on the list with 117 common nursing home conditions. Second, the number of hospitalizations that could have been prevented differed in case of optimal care conditions for the nursing home and outpatient setting, respectively. This may be due, in part, to the fact that Sundmacher $\mathrm{et} \mathrm{al}^{7}{ }^{7}$ estimated preventability for groups of ICD-10 conditions, clustered by disease category, while we estimated the preventability for every ICD-10 code individually. Although ICD-10 conditions and their estimated preventability differed in both health care settings, the main goal of these lists lies in raising awareness for which hospitalizations may be preventable.

The focus on nursing home-sensitive hospital admissions is therefore a critical issue for health care organization and reform. In addition to the financial implications associated with the potential of the diagnoses on our consensus list to avert hospitalization, reducing hospital admissions amongst nursing home residents would have a substantial impact on the person-centeredness of health care and quality of life of residents.

\section{Strengths and limitations}

A strength of our study is that we were able to combine the analysis of routine health insurance data with a two-stage Delphi study and expert workshop. The health insurance data covered nearly $30 \%$ of the statutory health insured persons in Germany. For the Delphi study, we were able to recruit a high-calibre expert group and succeeded in ensuring a very high response rate of over $90 \%$. Our subgroup analysis demonstrated that the assessment of experts was robust and not biased towards specialization, age or gender. This supports widespread recognition and applicability of our indicator list. By using the RAND/UCLA Appropriateness Method, ${ }^{22}$ enhancing the Delphi method with an expert workshop, we were able to introduce direct interaction between experts as in other consensus development methods, ${ }^{31}$ thereby combining the strengths of the Delphi methods with others.

The external validity of the results of the modified Delphi method, in general, is dependent on the representativeness of the panel of experts. The validity of the Delphi method depends, among other things, on the response rate, ${ }^{25}$ with response rates between $51 \%-80 \%$ being recommended in the literature. ${ }^{32-35}$ The commitment of participants to complete the Delphi process is often related to their interest and involvement with the question being examined. ${ }^{25}$ In our study, we observed a high intrinsic motivation of the experts to participate, as many waived the incentive offered, and as evidenced by the extremely high response rate of $91 \%$, with unlikely bias resulting from the minor loss-to follow-up. The validity of 
the Delphi method also depends on the included experts. ${ }^{26}$ The more diverse and heterogeneous the expert panel is, the higher the quality of the decisions. ${ }^{36}$ Four disciplines were represented in our panel, but no lay persons, such as nursing home residents or relatives. Another factor to consider when assessing the validity of the findings from the Delphi study is selection bias. If participants dropped out because of pseudonymity, there would only be a selection bias, if these individuals assessed the avoidance potential differently than the participants in this study. This is highly unlikely, as our results across Delphi rounds as well as various disciplines were very stable. The stability of the assessments in the individual Delphi rounds is considered more important than the response rate with regard to the occurrence of consensus. ${ }^{37}$ We observed specialty-specific statistically significant, though minimal and therefore irrelevant, differences in estimated hospitalization preventability after the second round of surveys, for a subset ( $n=34)$ of the 117 ICD-10 codes. Clinicians had slightly lower avoidability estimates, which could be due to the fact that they see the more serious cases in the clinic and are therefore more cautious with their assessment. Gender and age effects in estimating the proportion of potentially avoidable hospitalizations were low. Because of multidisciplinarity, the stable assessments of and the low dispersion in the estimates of the potential avoidability of hospitalization of nursing home residents and the relatively high number of included experts compared to other Delphi methods, it is rather unlikely that another panel would have come to different results. For these reasons, together with the high response rate, we expect the list of 58 nursing home-sensitive conditions to be generalizable.

The key limitation of our study, like comparable studies, is that preventability assessments were made assuming optimal structural and nursing home care conditions. We are aware that these conditions are currently not always met, and that interventions and improvement efforts are required to reduce or avoid hospital admission in the current health care setting.

\section{Researchers' characteristics and contextual factors}

All researchers were qualified health services researchers, familiar with the different methodological components of our study. However, the relationships between researchers and study participants were to a large extent limited to time-restricted conversations in a series of workshops. Given that participants were specialists invited for their specific expertise, researchers had no specific assumptions or presuppositions about the experts' input. In the manuscript, no further interpretations of the experts' inputs have been added.

The expert workshops and Delphi questionnaire - the only component of the research where qualitative comments were made - were all conducted online. No salient contextual factors impacting the research were identified.

\section{Implications for policy, research and clinical practice}

Our study has various implications for policy, practice and research. Similar to the research on ambulatory care-sensitive hospital admissions, we assume that our study will lead to substantial debate and controversy about variations on nursing home-sensitive hospital admission rates and on the policy response to this variation. Such debate is likely to lead to proposals for improvements of the organization of the health care delivery system, and to constitute new indicators to monitor health system performance. For all stakeholders (e.g., medical and nursing providers, policy makers, health economists), the list of nursing home-sensitive conditions can inform the development of interventions and facilitate local quality improvement efforts. Practitioners and researchers should collaborate to identify the type of interventions (including personnel, staffing, skill mix, continuing professional education, infrastructure/resources, technology) required to reduce hospital admissions. Research should further address a costing of these interventions and calculations on the headroom (the maximum cost of the intervention to be cost-effective) to inform managers of nursing homes, hospitals and delivery systems. Finally, internationally comparative research should aim to identify a robust core basket of indicators for nursing home-sensitive hospital conditions for different health care system contexts. ${ }^{20}$

\section{Data availability}

All data that can be directly shared has already been included in the manuscript. Further, we have (i) used relevant keywords and descriptions for other researchers to identify our research (Findable), (ii) described our routes to data access for other researchers to grant access to similar data (Accessible), (iii) have used international nomenclature (ICD-10 codes) to facilitate merging of our datasets with those of other researchers (Interoperable) and (iv) we encourage other researchers to build on and reuse our methodological approach and data for further exploitation of the research findings (Reusable).

\section{Underlying data}

Due to the provision of the data by the statutory health insurance (SHI) companies within the framework of a data evaluation contract, a direct publication is not possible. However, readers and reviewers may apply to access the data by contacting the following SHI companies. Several factors will be considered before access to data is granted, including the adherence to the EU General Data Protection Regulation. 
- General Local Health Insurance Fund (AOK) in Rhineland/Hamburg (aok@rh.aok.de)

- General Local Health Insurance Fund (AOK) Baden-Württemberg (info@bw.aok.de)

- General Local Health Insurance Fund (AOK) Rhineland-Palatinate/Saarland (service@ rps.aok.de)

- BARMER Health Insurance Fund (service@barmer.de)

- German Employees' Health Insurance Fund (DAK; service@ dak.de)

- Health Insurance Fund (BKK) Werra-Meissner (info@bkk-wm.de)

Extended data

Open Science Framework: "Nursing home-sensitive conditions", https://doi.org/10.17605/OSF.IO/EAJ58 38

This project contains the following extended data:

- f1000Extended data_2021-11-03.pdf (questionnaire, workshop and data request documentation)

Data are available under the terms of the Creative Commons Attribution 4.0 International license (CC-BY 4.0).

\section{Acknowledgements}

We thank researchers and postgraduate students at the Chair of Management and Innovation in Health Care at the Witten/ Herdecke University, Germany; Prof. Dr. Hagen Bachmann, Chair of Pharmacology and Toxicology at the Witten/ Herdecke University, Germany; Prof. Dr. Petra Thürmann, Chair of Clinical Pharmacology at the Witten/Herdecke University, Germany; Prof. Dr. Hans-Jürgen Heppner, Chair of Geriatrics at the Witten/Herdecke University, Germany; Prof. Dr. Thomas Klie, FIVE- Registered Association for Research and Innovation of the Protestant University of Applied Sciences Freiburg, Research focus group Social Research in Gerontology and Nursing, Freiburg, Germany; Dr. honoris causa Helmut Hildebrandt, OptiMedis Inc., Hamburg, Germany; Prof. Dr. Christel Bienstein, Registered Association for Nursing, Berlin, Germany; Prof. Dr. Andreas Sönnichsen, Department of General Practice and Family Medicine, Centre for Public Health at the Medical University of Vienna, for their academic support. We also thank Philip Lewin, OptiMedis Inc., Hamburg, Germany, for his support in programming the online tools.

We acknowledge the following German statutory health insurance companies, for providing the anonymized hospital release data on nursing home residents: General Local Health Insurance Fund (AOK) in Rhineland/Hamburg, BadenWürttemberg, Rhineland-Palatinate/Saarland, BARMER Health Insurance Fund, German Employees' Health Insurance Fund (DAK), and the Health Insurance Fund (BKK) Werra-Meissner.

Last but not least, we would like to thank all ambulatory and clinical physicians, nurses, scientists, and other experts who, despite the COVID-19 pandemic, took the time to participate in both lengthy surveys and/or workshop for the adjusted Delphi process.

1. Palleschi L, De Alfieri W, Salani B, et al.: Functional recovery of elderly patients hospitalized in geriatric and general medicine units. The PROgetto DImissioni in GEriatria Study.J. Am. Geriatr. Soc. 2011; 59(2): 193-199.

PubMed Abstract | Publisher Full Text

2. Page DB, Donnelly JP, Wang HE: Community-, Healthcare-, and Hospital-Acquired Severe Sepsis Hospitalizations in the
University Health System Consortium. Crit. Care Med. 2015; 43(9): 1945-1951.

PubMed Abstract | Publisher Full Text | Free Full Text

3. O'Hara DA, Carson NJ: Reporting of adverse events in hospitals in Victoria, 1994-1995. Med. J. Aust. 1997; 166(9): 460-463. Publisher Full Text
4. Palese A, Gonella S, Moreale R, et al.: Hospital-acquired functional decline in older patients cared for in acute medical wards and predictors: Findings from a multicentre longitudinal study. Geriatr. Nurs. 2016; 37(3): 192-199.

PubMed Abstract | Publisher Full Text

5. Purdy S, Griffin T, Salisbury C, et al.: Ambulatory care sensitive conditions: terminology and disease coding need to be more specific to aid policy makers and clinicians. Public Health. 2009; 123(2): 169-173.

PubMed Abstract | Publisher Full Text

6. Purdy S, Paranjothy S, Huntley A, et al.: Interventions to reduce unplanned hospital admission: a series of systematic reviews. Bristol, 
Great Brittain: National Institute for Health Research. 2012 June 2012. Report No.: Grant PB-PG-120818013.

7. Sundmacher L, Fischbach D, Schuettig W, et al.: Which hospitalisations are ambulatory care-sensitive, to what degree, and how could the rates be reduced? Results of a group consensus study in Germany. Health Policy (Amsterdam, Netherlands). 2015; 119(11): 1415-1423. PubMed Abstract | Publisher Full Text

8. Ouslander JG, Lamb G, Perloe M, et al.: Potentially avoidable hospitalizations of nursing home residents: frequency, causes, and costs. J. Am. Geriatr. Soc. 2010; 58(4): 627-635. PubMed Abstract| Publisher Full Text

9. Freund T, Heller G, Szecsenyi J: Krankenhausfälle für ambulant behandelbare Erkrankungen in Deutschland. Z. Evid. Fortbild. Qual. Gesundhwes. 2014; 108: 251-257. Publisher Full Text

10. Leutgeb R, Berger SJ, Szecsenyi J, et al.: Potentially avoidable hospitalisations of German nursing home patients? A crosssectional study on utilisation patterns and potential consequences for healthcare. BMJ Open. 2019; 9(1): e025269. PubMed Abstract | Publisher Full Text | Free Full Text

11. Intrator $O$, Zinn J, Mor V: Nursing home characteristics and potentially preventable hospitalizations of long-stay residents. J. Am. Geriatr. Soc. 2004; 52(10): 1730-1736. PubMed Abstract | Publisher Full Text

12. Hsieh VC, Hsieh ML, Chiang JH, et al.: Emergency Department Visits and Disease Burden Attributable to Ambulatory Care Sensitive Conditions in Elderly Adults. Sci. Rep. 2019; 9(1): 3811. PubMed Abstract | Publisher Full Text | Free Full Text

13. McAndrew RM, Grabowski DC, Dangi A, et al.: Prevalence and patterns of potentially avoidable hospitalizations in the US long-term care setting. International journal for quality in health care: journal of the International Society for Quality in Health Care. 2016; 28(1): 104-109. PubMed Abstract | Publisher Full Text

14. Carter MW: Factors associated with ambulatory care-sensitive hospitalizations among nursing home residents. J. Aging Health 2003; 15(2): 295-331.

PubMed Abstract | Publisher Full Text

15. World Health Organisation: Assessing health services delivery performance with hospitalizations for ambulatory care sensitive conditions. Working document. Copenhagen, Denmark. 2016.

16. Spector WD, Limcangco R, Williams $C$, et al.: Potentially avoidable hospitalizations for elderly long-stay residents in nursing homes. Med. Care. 2013; 51(8): 673-681. PubMed Abstract | Publisher Full Text

17. Walker JD, Teare GF, Hogan DB, et al.: Identifying potentially avoidable hospital admissions from canadian long-term care facilities. Med. Care. 2009; 47(2): 2504.

18. Walsh EG, Wiener JM, Haber $S$, et al.: Potentially avoidable hospitalizations of dually eligible Medicare and Medicaid beneficiaries from nursing facility and Home- and CommunityBased Services waiver programs. J. Am. Geriatr. Soc. 2012; 60(5): 821-829.

PubMed Abstract | Publisher Full Text

19. Walsh EG, Freiman M, Haber SG, et al.: Cost Drivers for Dually Eligible Beneficiaries: Potentially Avoidable Hospitalizations from Nursing Facility, Skilled Nursing Facility, and Home and Community-Based Services Waiver Programs. Washington DC, U.S.A.: Research Triangle Institute (RTI International); 2010 August 2010. Report No.: RTI Project Number 0209853.022 Contract No.: CMS Contract No. HHSM-500-2005-00029I.

20. Allers K, Hoffmann F, Schnakenberg R: Hospitalizations of nursing home residents at the end of life: A systematic review. Palliat. Med. 2019; 33(10): 1282-1298.

PubMed Abstract | Publisher Full Text | Free Full Text
21. Swart E, Gothe H, Geyer S, et al.: Good Practice of Secondary Data Analysis (GPS): Guidelines and Recommendations. Gesundheitswesen (Bundesverband der Arzte des Offentlichen Gesundheitsdienstes (Germany)). 2015; 77: 120-126. PubMed Abstract | Publisher Full Text

22. Fitch K, Bernstein SJ, Aguilar MD, et al.: The RAND/UCLA Appropriateness Method User's Manual. Santa Monica, California, USA: RAND Corporation; 2001. Report No.: MR-1269-DG-XII/RE

23. Schulte T, Schwab T, Dittmann B: Analyse pflegebedürftiger Patienten relativ zum Zeitpunkt des Todes. Rebscher $\mathrm{H}$, editor. Beiträge zur Gesundheitsökonomie und Versorgungsforschung. Band 14 Heidelberg, Germany: medhochzwei; 2016; p. 43-67.

24. Donohoe H, Needham R: Moving Best Practice Forward: Delphi Characteristics, Advantages, Potential Problems, and Solutions. Int. J. Tour. Res. 2009; 11: 415-437. Publisher Full Text

25. Hasson $F$, Keeney $S$, McKenna $H$ : Research guidelines for the Delphi survey technique. J. Adv. Nurs. 2000; 32(4): 1008-1015. PubMed Abstract

26. Okoli C, Pawlowski SD: The Delphi method as a research tool: an example, design considerations and applications. Inf. Manag. 2004; 42(1): 15-29. Publisher Full Text

27. Mayring P: Qualitative content analysis: theoretical foundation, basic procedures and software solution. Klagenfurt: 2014.

28. Destatis: Pflegestatistik. Pflege im Rahmen der Pflegeversicherung. Deutschlandergebnisse. Wiesbaden: Statistisches Bundesamt (Destatis); 2018.

29. Bundesministerium für Gesundheit: Ausbau ambulanter Wohnund Betreuungsangebote für Menschen im Alter, mit Pflegebedürftigkeit oder Behinderung Berlin/Köln: Bundesanzeiger Verlag GmbH. 2020. [Drucksache 19/23342, Antwort der Bundesregierung]. Reference Source

30. Organisation for Economic Cooperation and Development (OECD): Demographic References: Population age structure 2021. Reference Source

31. Black N, Murphy M, Lamping D, et al.: Consensus development methods: a review of best practice in creating clinical guidelines. J. Health Serv. Res. Policy. 1999; 4(4): 236-248. PubMed Abstract | Publisher Full Text

32. Green $B$, Jones $M$, Hughes $D$, et al.: Applying the Delphi technique in a study of GPs' information requirements. Health Soc. Care Community. 1999; 7(3): 198-205

PubMed Abstract | Publisher Full Text

33. Loughlin KG, Moore LF: Using Delphi to achieve congruent objectives and activities in a pediatrics department.J. Med. Educ. 1979; 54(2): 101-106.

PubMed Abstract | Publisher Full Text

34. McKenna H: The Delphi technique: A worthwhile research approach for nursing?. J. Adv. Nurs. 1994; 19: 1221-1225. PubMed Abstract | Publisher Full Text

35. Sumsion T: The Delphi Technique: An Adaptive Research Tool. Br. J. Occup. Ther. 1998; 61(4): 153-156. Publisher Full Text

36. Powell C: The Delphi technique: myths and realities. J. Adv. Nurs. 2003; 41(4): 376-382. PubMed Abstract | Publisher Full Text

37. Crisp J, Pelletier D, Duffield C, et al.: The Delphi Method?. Nurs. Res. 1997; 46(2): 116-118. PubMed Abstract | Publisher Full Text

38. Bohnet-Joschko S, Valk-Draad MP, Schulte T, et al.: Nursing homesensitive conditions. 2021.

Publisher Full Text 


\section{Open Peer Review}

\section{Current Peer Review Status:}

\section{Version 1}

Reviewer Report 21 March 2022

https://doi.org/10.5256/f1000research.77561.r126098

(C) 2022 Badgery-Parker T. This is an open access peer review report distributed under the terms of the Creative Commons Attribution License, which permits unrestricted use, distribution, and reproduction in any medium, provided the original work is properly cited.

\section{Tim Badgery-Parker}

Australian Institute of Health Innovation, Faculty of Medicine, Health and Human Sciences, Macquarie University, Sydney, NSW, Australia

This article describes the development of a list of "nursing home sensitive" conditions, and the use of the list to estimate costs of potentially preventable admissions from nursing homes to hospitals in Germany. The authors demonstrate differences between their list and an existing list of ambulatory sensitive conditions, confirming that the nursing home setting is sufficiently different, that the use of ambulatory care sensitive conditions is unlikely to be appropriate.

The candidate diagnoses are derived from a large administrative dataset ( $30 \%$ of nursing home residents in Germany) and the process of developing the list is appropriate and comprehensively described, as is the cost estimation. The language is generally quite good, with some stray punctuation and a few unclear sentences.

The results include a comparison of the preventability estimates between subgroups of the Delphi panel, this does not appear to be described in the methods.

The results also describe criteria for excluding or combining diagnoses after the first round for presentation in the second round. (The criteria were not met for any diagnosis.) These criteria should really be in the methods.

Overall this is a strong study that makes a valuable contribution to the field.

Is the work clearly and accurately presented and does it cite the current literature? Partly

Is the study design appropriate and is the work technically sound?

Yes

Are sufficient details of methods and analysis provided to allow replication by others? 
Yes

If applicable, is the statistical analysis and its interpretation appropriate?

Yes

Are all the source data underlying the results available to ensure full reproducibility? Yes

Are the conclusions drawn adequately supported by the results?

Yes

Competing Interests: No competing interests were disclosed.

Reviewer Expertise: I am a biostatistician with considerable experience in using administrative data to examine low-value hospital care and patient safety.

I confirm that I have read this submission and believe that I have an appropriate level of expertise to confirm that it is of an acceptable scientific standard.

\section{Author Response 01 Apr 2022}

\section{Sabine Bohnet-Joschko}

Dear editors, Dear Tim Badgery-Parker,

We kindly thank you for your reply and for offering us the revision of our manuscript for consideration in the F1000Research Platform. We carefully considered the comments offered by you to improve the quality of the initial draft of the manuscript.

Please find below a point-by-point response, in which we describe how we have addressed the suggestions. All changes or adjustments are shown in italic font and in revision mode in the revised paper accordingly.

Again thank you for taking the time to critically review the paper. We truly appreciate the constructive input and hope that our revisions meet your approval.

Yours sincerely, from the research team,

Prof. Dr. Sabine Bohnet-Joschko

\section{Comment 1:}

"The results include a comparison of the preventability estimates between subgroups of the Delphi panel, this does not appear to be described in the methods."

\section{Authors' response to comment 1:}

Thank you very much indeed for your review and the comments.

We have now added two sentences to address this aspect:

In the "Method" section, subsection "Delphi study and expert workshop", fourth paragraph, last sentence: 
“...and planned to recruit 100 experts: 30 outpatient/clinical physicians each, 30 nursing professionals and 10 scientists. This also allowed for comparison of preventability assessments by expert group."

And in the ninth paragraph:

"In case the preventability potential of at least $75 \%$ was not comprised in the range of $75 \%$ of all expert assessments, the ICD-10 codes were excluded. For all other ICD-10 codes, statistical data (median, modal values, dispersion parameters, bar charts), results of expert group comparison on preventability assessments (Kruskal-Wallis (K-W)-Test), and categorized comments from the Delphi questionnaire were provided for the expert workshop."

\section{Comment 2:}

"The results also describe criteria for excluding or combining diagnoses after the first round for presentation in the second round. (The criteria were not met for any diagnosis.) These criteria should really be in the methods."

\section{Authors' response to comment 2:}

Thank you for bringing this to our attention, which we, unfortunately, overlooked before. We have now moved the criteria to the methods section and only referred to them in the results section, as follows:

In the "Method" section, subsection "Delphi study and expert workshop", eighth paragraph, second sentence:

"ICD-10 codes were planned to be excluded for assessment in the second round, when at least $75 \%$ of the experts estimated the preventability as zero in the first round. Secondly, ICD-10 codes were planned to be combined if the diseases were very similar (in terms of symptoms, diagnosis, prognosis, treatment) and the proportions of potential preventability were nearly identical. Two conditions for "nearly identical potential preventability" had to be fulfilled: the medians of the estimated preventability should not differ by more than $5 \%$ and the limits of the interquartile range had to be less than 10\% apart. Based on this information, the ICD-10 codes for the second round were identified and the participants were asked to quantify the proportion of potentially avoidable hospitalization again in the second round."

And in the "Results" section, subsection "Delphi study and expert workshop", second paragraph, rather at the end, we changed the text "Furthermore, ICD-10 Codes... . Neither conditions were met sufficiently." was changed as follows:

"The condition that at least $75 \%$ of the experts estimated the preventability as zero in the first round was met for none of the ICD-10 codes. Furthermore, none of the ICD-10 codes could be combined as the conditions for "nearly identical potential preventability" were not fulfilled."

Competing Interests: No competing interests were disclosed. 
Reviewer Report 24 February 2022

https://doi.org/10.5256/f1000research.77561.r119120

(C) 2022 Holle D. This is an open access peer review report distributed under the terms of the Creative Commons Attribution License, which permits unrestricted use, distribution, and reproduction in any medium, provided the original work is properly cited.

\section{Daniela Holle it}

Hochschule für Gesundheit, University of Applied Sciences, Bochum, Germany

Thank you for the option to review the manuscript entitled "Nursing home-sensitive conditions: analysis of routine health insurance data and modified Delphi analysis of potentially avoidable hospitalizations".

In a first research question, the study examines how often nursing home residents are treated in hospitals and what are the main diagnosis and associated costs of these hospital admissions. The second research question addresses potentially avoidable hospitals admission of nursing home residents. The third research question asks for the impact of the estimated preventability of hospital admissions. To answer the questions a secondary analysis of routine data based on health insurance claims data were used. In addition, a Delphi survey in combination with an expert workshop was conducted.

In view of rising healthcare costs and increasingly scarce healthcare resources, answering the research questions is essential to ensure the best possible patient care while maintaining patient safety. A striking feature of the study is the very large data set of approximately $€ 240,000$ nursing home residents, which includes one-third of all statutorily insured nursing home residents. This is particularly noteworthy with regard to the representativeness of the study finding. The large number of experts who participated in the Delphi survey should also be emphasized.

On closer inspection, however, I noticed a few aspects that I would like to briefly outline here: The introduction notes that interventions to reduce hospitalizations of nursing home residents should be tailored to health care systems. As an example of possible interventions, studies on the introduction and testing of nurse-led care models in nursing homes could be listed here. For example, the recent study by Zuniga et al (see Zúñiga et al. Positive effect of the INTERCARE nurseled model on reducing nursing home transfers: A nonrandomized stepped-wedge design. ${ }^{1}$ ) demonstrates that with an introduction of a nurse-led care model to hospital admissions of nursing home residents can be significantly reduced. Similar positive approaches can be found, for example, on the recent scoping review by Schmüdderich et al. (see Schmüdderich et al. Core elements and potential of nurse-led care models in residential long-term care: A scoping review. J Clin Nurs. 2022 Feb 4. ${ }^{2}$ ). These efforts in the reduction of hospital admissions from nursing homes are worth noticing in the introduction.

The methodology of the study is presented in a structured and comprehensible manner. However, from a content perspective, the study provides some limitations that I would like to outline briefly here and that should be critically presented and discussed in the course of the study. The routine data on discharge diagnoses were used to describe the admission diagnosis of 
nursing home residents. It should be critically reflected that there is a process between admission and discharge of a hospital patient, which can lead to confirmation, extension, or refutation of the admission diagnosis. Thus, the admission diagnosis is a limited indicator to describe the frequency and nature of hospital admissions of nursing home residents.

With regard to the avoidability of hospital admissions, it must also be taken into account that the admission diagnosis alone is not necessarily the decisive factor for a corresponding admission, but also resident-specific criteria, which, in combination with a diagnosis, can lead to hospitalization. Thus, hospitalization may not be avoidable even with optimal care due to specific characteristics of nursing home residents.

In this study, the significance of the results on the estimated avoidability of hospital admission mainly depends on the definition of optimal care for nursing home residents. In this manuscript, optimal care is defined by access to trained personnel, resources, and infrastructure for monitoring and nursing, and cooperation agreements with ambulatory general and specialists care providers (page 4/29, manuscript). Further explanations for the definition of optimal care are given within the attachment of the extended data (page 21/359):

well-trained nursing staff

the possibility or the equipment for a preliminary examination, for monitoring a possible crisis in the condition of the nursing home resident and for initiating treatment measures good cooperation with outpatient medical care

the possibility of consultation with a specialist

In the definition of optimal care, it must be critically appreciated that both the qualification of the nursing staff and the cooperation of different professions were included, although well-trained outpatient medical care staff is also needed for optimal care.

Nevertheless, the criteria for optimal care are very general, which is why a later discussion on the impact of avoidable hospital admissions also proves to be very difficult. With regard to the nursing staffing, the ratio between residents and nursing staff is relevant on the one hand, but also the skill and grade mix of the nursing staff against the background of the current resident structure in nursing homes. A good cooperation with the outpatient medical care could also have been specified in more detail, e.g. how often the individual resident is visited by an outpatient medical care service, how often the resident is also cared for by a specialist.

The more specifically optimal care would have been described here, the more specifically the experts' assessments could have been estimated and the costs for the expenditure of optimal care could have been calculated and compared with the costs for preventable hospital admissions. In the course of the conclusion, it is stated that interventions should be developed that contribute to the reduction of hospitalizations of nursing home residents. In this context, it would be useful to refer to existing concepts (see comment introduction).

I hope my comments help to strengthen the manuscript.

\section{References}

1. Zúñiga F, Guerbaai RA, de Geest S, Popejoy LL, et al.: Positive effect of the INTERCARE nurse-led model on reducing nursing home transfers: A nonrandomized stepped-wedge design.J Am Geriatr Soc. 2022. PubMed Abstract | Publisher Full Text 
2. Schmüdderich K, Kiwitt J, Palm R, Roes M, et al.: Core elements and potential of nurse-led care models in residential long-term care: A scoping review.J Clin Nurs. 2022. PubMed Abstract |

Publisher Full Text

Is the work clearly and accurately presented and does it cite the current literature? Partly

Is the study design appropriate and is the work technically sound? Partly

Are sufficient details of methods and analysis provided to allow replication by others? Yes

If applicable, is the statistical analysis and its interpretation appropriate? Yes

Are all the source data underlying the results available to ensure full reproducibility? Partly

Are the conclusions drawn adequately supported by the results? Partly

Competing Interests: No competing interests were disclosed.

Reviewer Expertise: My focus is on nursing research, particularly in the area of nursing home care. I have no expertise in conducting economic studies.

I confirm that I have read this submission and believe that I have an appropriate level of expertise to confirm that it is of an acceptable scientific standard, however I have significant reservations, as outlined above.

\section{Author Response 01 Apr 2022}

\section{Sabine Bohnet-Joschko}

Dear Editors, Dear Daniela Holle,

We kindly thank you for your reply and for offering us the revision of our manuscript for consideration in the F1000Research Platform. We carefully considered the comments offered by you to improve the quality of the initial draft of the manuscript.

Please find below a point-by-point response, in which we describe how we have addressed the suggestions. All changes or adjustments are shown in italic font in the table below and in track changing mode in the revised paper accordingly.

Again thank you for taking the time to critically review the paper. We truly appreciate the constructive input and hope that our revisions meet your approval. 
Yours sincerely, from the research team,

Prof. Dr. Sabine Bohnet-Joschko

\section{Comment 1:}

"As an example of possible interventions, studies on the introduction and testing of nurseled care models in nursing homes could be listed here. For example, the recent study by

Zuniga et al (see Zúñiga et al. Positive effect of the INTERCARE nurse-led model on reducing nursing home transfers: A nonrandomized stepped-wedge design.) demonstrates that with an introduction of a nurse-led care model to hospital admissions of nursing home residents can be significantly reduced. Similar positive approaches can be found, for example, on the recent scoping review by Schmüdderich et al. (see Schmüdderich et al. Core elements and potential of nurse-led care models in residential long-term care: A scoping review. J Clin Nurs. 2022 Feb 4.). These efforts in the reduction of hospital admissions from nursing homes are worth noticing in the introduction."

\section{Authors' response to comment 1:}

Thank you for this valuable comment. We have now added the following sentence in the "Introduction" section, at the end of the second to last paragraph, and incorporated both references:

"For instance, nurse-led care models with higher qualified nurses in expanded roles have been introduced and showed a positive impact on reducing hospitalization of nursing home residents and on advancing nursing practice in nursing homes, (Zúñiga et al. (2022), Schmüdderich et al. (2022))."

\section{Comment 2:}

"The routine data on discharge diagnoses were used to describe the admission diagnosis of nursing home residents. It should be critically reflected that there is a process between admission and discharge of a hospital patient, which can lead to confirmation, extension, or refutation of the admission diagnosis. Thus, the admission diagnosis is a limited indicator to describe the frequency and nature of hospital admissions of nursing home residents."

\section{Authors' response to comment 2:}

Thank you for this comment: You are absolutely right, and therefore our study was based on discharge diagnoses of the nursing home residents instead of on admission diagnoses.

To clarify this, we have added the following sentence to the "Methods" section, subsection "Analysis of routine health insurance data", at the end of the second paragraph:

"In line with previous studies, our analysis of routine health insurance data focused on the hospital discharge diagnosis, not on the admission diagnosis, as the latter is often subject to confirmation, extension or refutation during the hospital stay."

\section{Comment 3:}

"With regard to the avoidability of hospital admissions, it must also be taken into account that the admission diagnosis alone is not necessarily the decisive factor for a corresponding 
admission, but also resident-specific criteria, which, in combination with a diagnosis, can lead to hospitalization. Thus, hospitalization may not be avoidable even with optimal care due to specific characteristics of nursing home residents."

\section{Authors' response to comment 3:}

This is absolutely correct and is well reflected by nursing home-sensitive conditions only expected to be avoidable in at least $70 \%$ of all cases with this condition. Conversely, this automatically means that up to $30 \%$ of the remaining cases with a disease represented in the nursing home-sensitive conditions catalogue are unavoidable hospitalizations.

We included the following sentence under "Key findings" to clarify this:

"After two Delphi rounds and an expert workshop, we were able to identify 58 diagnoses considered to be nursing home-sensitive conditions in the context of the German health care system, i.e., at least seventy percent of all hospitalizations with such a condition is expected to be preventable, depending on the individual health status and advance directive of the nursing home resident."

\section{Comment 4:}

In the definition of optimal care, it must be critically appreciated that both the qualification of the nursing staff and the cooperation of different professions were included, although well-trained outpatient medical care staff is also needed for optimal care.

Nevertheless, the criteria for optimal care are very general, which is why a later discussion on the impact of avoidable hospital admissions also proves to be very difficult. With regard to the nursing staffing, the ratio between residents and nursing staff is relevant on the one hand, but also the skill and grade mix of the nursing staff against the background of the current resident structure in nursing homes. A good cooperation with the outpatient medical care could also have been specified in more detail, e.g. how often the individual resident is visited by an outpatient medical care service, how often the resident is also cared for by a specialist.

The more specifically optimal care would have been described here, the more specifically the experts' assessments could have been estimated and the costs for the expenditure of optimal care could have been calculated and compared with the costs for preventable hospital admissions.

\section{Authors' response to comment 4:}

Thank you for this detailed evaluation of this aspect of our study. All mentioned aspects do matter indeed. We had some practical restraints, that made us decide to define optimal care conditions the way we did, which we have explained now in more detail in the "Discussion" section under "Strengths and limitations":

"The description of optimal care conditions was rather short, and perhaps therefore rather general. This could have led to less accurate preventability estimates. By addressing optimal care conditions in more detail (e.g., the importance of well-trained nursing staff both in the out-as well as the inpatient setting, special geriatric care knowledge and skills, resident structure in the nursing home, resident/ nursing staff ratio, skill and grade mix of staff, good cooperation with outpatient medical care, frequency of medical care visits by an outpatient medical care servicel 
specialist), experts might have given other (higher) estimates of preventability. This might have influenced the extrapolation of costs as well. As we already had a very long list of instructions, explanations and conditions to be assessed, we decided not to overstrain clarity and longevity for the Delphi experts hereupon, and kept the information provided on optimal care conditions to the point."

\section{Comment 5:}

In the course of the conclusion, it is stated that interventions should be developed that contribute to the reduction of hospitalizations of nursing home residents. In this context, it would be useful to refer to existing concepts (see comment introduction).

\section{Authors' response to comment 5:}

We addressed this significant input in the "Discussion" section under "Implications for policy, research and clinical practice" as follows:

"For all stakeholders (e.g., medical and nursing providers, policy makers, health economists), the list of nursing home-sensitive conditions can inform the development of interventions and facilitate local quality improvement efforts, also taking into account existing evidence-based concepts."

Competing Interests: No competing interests were disclosed.

The benefits of publishing with F1000Research:

- Your article is published within days, with no editorial bias

- You can publish traditional articles, null/negative results, case reports, data notes and more

- The peer review process is transparent and collaborative

- Your article is indexed in PubMed after passing peer review

- Dedicated customer support at every stage

For pre-submission enquiries, contact research@f1000.com 